\title{
Ubiquitin Extension Protein UEP1 Modulates Cell Death and Resistance to Various Pathogens in Tobacco
}

\author{
You-Ping Xu, ${ }^{1,2,3}$ Yuan Zhao, ${ }^{2}$ Xiao-Yi Song, ${ }^{2}$ Yun-Feng Ye, ${ }^{2}$ Ren-Gang Wang, ${ }^{1}$ Zi-Li Wang, ${ }^{1}$ Xue-Liang Ren, ${ }^{1, \dagger}$ \\ and Xin-Zhong Cai ${ }^{2, \dagger}$ \\ ${ }^{1}$ Molecular Genetics Key Laboratory of China Tobacco (Guizhou Academy of Tobacco Science), Guiyang 550081, China \\ ${ }^{2}$ Institute of Biotechnology, College of Agriculture and Biotechnology, Zhejiang University, Hangzhou 310058, China \\ ${ }^{3}$ Centre of Analysis and Measurement, Zhejiang University, Hangzhou 310058, China \\ Accepted for publication 27 March 2019.
}

ABSTRACT

\begin{abstract}
Ubiquitin (Ub) extension proteins (UEPs) are fusion proteins of a Ub at the $\mathrm{N}$ terminus to a ribosomal protein. They are the main source of $\mathrm{Ub}$ and the only source of extension ribosomal protein. Although important roles of the Ub-26S proteasome system in various biological processes have been well established, direct evidence for the role of $U E P$ genes in plant defense is rarely reported. In this study, we cloned a Ub-S27a-type UEP gene (NbUEP1) from Nicotiana benthamiana and demonstrated its function in cell death and disease resistance. Virus-induced gene silencing of $N b U E P 1$ led to intensive cell death, culminating in wholeseedling withering. Transient RNA interference (RNAi) of NbUEP1 caused strong cell death in infiltrated areas, while stable NbUEPI-RNAi tobacco plants constitutively formed necrotic lesions in leaves. NbUEP1-RNAi plants exhibited increased resistance to the oomycete
\end{abstract}

Pythium aphanidermatum and viruses Tobacco mosaic virus and Cucumber mosaic virus while displaying decreased resistance to the nematode Meloidogyne incognita compared with non-RNAi control plants. Transcription profiling analysis indicated that jasmonate and ethylene pathways, lipid metabolism, copper amine oxidase-mediated active species generation, glycine-rich proteins, vacuolar processing enzyme- and RD21-mediated cell death and defense regulation, and autophagy might be associated with $N b U E P 1$-mediated cell death and resistance. Our results provided evidence for the important roles of plant UEPs in modulating plant cell death and disease resistance.

Keywords: cell death, disease resistance, genetics and resistance, tobacco, ubiquitin, ubiquitin extension protein
Ubiquitin (Ub), comprising 76 amino acids (aa), is a highly conserved peptide in eukaryotes. Ubiquitylation, commonly mediated by a Ub-activating enzyme (E1), a Ub-conjugating enzyme (E2), and a Ub ligase enzyme (E3), is a well established system of posttranslational modification (Vierstra 2012). The ubiquitylated proteins are generally degraded by $26 \mathrm{~S}$ proteasome. The Ub-26S proteasome system is widely involved in regulation of various biological processes by targeting key regulatory proteins of these processes. Over the past years, the important roles of this pathway in plant-pathogen interactions have also been increasingly unveiled. The Ub-26S proteasome system contributes to a variety of defense mechanisms in plants against various pathogens, including the tiered plant defense system comprising pathogen-associated molecular pattern (PAMP)triggered immunity (PTI) and effector-trigged immunity (ETI), while pathogens inhibit or usurp this system to favor their infection and disease development (Marino et al. 2012; Zhou and Zeng 2017).

$\mathrm{Ub}$ exists as $\mathrm{Ub}$ polymer or $\mathrm{N}$-terminal $\mathrm{Ub}$ fusion to ribosomal proteins, the later being recognized as Ub extension proteins (UEPs) (Callis et al. 1995). Based on the fused ribosomal protein, the

†Corresponding authors: X.-Z. Cai; xzhcai@zju.edu.cn; and X.-L. Ren; renxuel@126.com

Funding: This work was financially supported by grants from the Zhejiang Provincial Natural Science Foundation of China (LZ18C140002, R307396), the National Natural Science Foundation of China (31672014) and the Open Foundation of Molecular Genetics Key Laboratory of China Tobacco (Guizhou Academy of Tobacco Science) (CNTC-[2014]334-No.110201302018 and CNTC[2015]346-No.110201503007)

*The $\boldsymbol{e}$-Xtra logo stands for "electronic extra" and indicates that two supplementary figures and two supplementary tables are published online.

The author(s) declare no conflict of interest.

(c) 2019 The American Phytopathological Society currently known UEPs are divided into two types: ribosomal L40type and ribosomal S27A-type. The two classes of ribosomal proteins differ in length and gene structure. The ribosomal L40 fused to $\mathrm{Ub}$ is approximately 52 aa in length and this type of $U E P$ gene contain introns in the coding region, whereas the ribosomal S27A fused to $\mathrm{Ub}$ is approximately 81 aa in length and the corresponding $U E P$ genes do not contain intron in the coding region (Callis et al. 1990). Functions of UEP genes in plants are still not fully understood. As in other higher eukaryotes, the initial translation products of Arabidopsis UEP genes are processed to separate the extension protein from Ub (Callis et al. 1990) by cleaving by Ub-specific proteases (Yan et al. 2000). The UEPs are not only the unique source for the extension ribosomal proteins but also the most important source for Ub. The extension ribosomal protein moiety of Arabidopsis UEP proteins is constituent of ribosomes, while the Ub moiety may act as a chaperone and assist in the formation of the holoribosome, as observed for other higher eukaryotes (Finley et al. 1989; Redman and Rechsteiner 1989; Spence et al. 2000). A study for a Ub-S27a-type UEP from Nicotiana benthamiana demonstrated that the Ub moiety did not affect the cellular localization of S27a but did alter its protein level, because expression of S27a without the Ub moiety caused a severe reduction in S27a protein level (Hanania et al. 2009). Additionally, the mature form of Ub released from UEPs after proteolysis may function as typical Ub in protein modification and, thus, is involved in various biological processes. Little is known about the function of extension ribosomal protein moiety. Given that they are assembled into ribosomes, they may be involved in protein biosynthesis. In addition, the ribosomal protein S27a of a UEP from $N$. benthamiana plays a critical role in the meristematic cell differentiation and proliferation. Overexpression of this S27a was correlated with increased proliferation of undifferentiated cells and arrest of morphologically "normal" shoot and leaf development (Hanania et al. 2009). 
Although the important roles of the Ub-26S proteasome system in plant development and plant-pathogen interactions have been well established, the direct evidence for the role of $U E P$ genes in plant-pathogen interactions is rarely reported. The potato cyst nematode Globodera rostochiensis effector GrUBCEP12 is a UEP that consists of a signal peptide for secretion, a monoubiquitin domain, and a 12-aa carboxyl extension protein (CEP12) domain that does not have similarity to any known protein. This secreted noncanonical UEP effector is involved in its plant parasitism. When delivered into host plant cells, this UEP is processed into free Ub and a CEP12 peptide (GrCEP12) in planta, and GrCEP12 suppresses resistance-gene-mediated cell death and basal resistance against the nematode $G$. rostochiensis, an unrelated bacterial pathogen (Streptomyces scabies) (Chronis et al. 2013), and flg22mediated PTI (Chen et al. 2013). Moreover, this UEP but not GrCEP12 potentially affects the host $26 \mathrm{~S}$ proteasome to promote feeding cell formation (Chronis et al. 2013). Nevertheless, to date, the direct evidence for the function of plant UEP in disease resistance is still lacking.

In this study, we demonstrated the function of a tobacco Ub-S27atype $U E P$ gene in cell death and disease resistance, and analyzed the possible mechanisms underlying these functions. Our results provided evidence for the important roles of plant UEP in modulating cell death and disease resistance.

\section{MATERIALS AND METHODS}

Cloning and sequence analyses of $N b U E P 1$. $N b U E P 1$ was cloned from $N$. benthamiana by real-time polymerase chain reaction (RT-PCR). Total RNA was extracted with TRIzol (Invitrogen), and reverse transcription from total RNA was conducted using the PrimeScript RT-PCR kit (TaKaRa Biotechnology). PCR was then performed from cDNAs using gene-specific primer pair NbUbi-F/ NbRlp-R, designed according to the full-length cDNA sequence of the UEP (AY912494) (Supplementary Table S1). PCR products were ligated into T-vector and sequenced. The cloned sequence was compared with the other tobacco UEP sequences, including the $N$. tabacum UEP gene (AJ223329) and N. benthamiana UEP gene (AY912494) using GeneDoc program (Nicholas et al. 1997).

Virus-induced gene silencing assay. Virus-induced gene silencing (VIGS) analyses and their efficiency evaluation were conducted as described previously (Li et al. 2012). A 383-bp sequence of $N b U E P 1$, including both $\mathrm{Ub}$ and ribosomal protein moieties, was amplified with primers NbUEP-F3 and NbUEP-R3 and inserted into Tobacco rattle virus (TRV)-based VIGS vector pYL156 with the sites EcoRI and BamHI. The recombinant pYL156-NbUEP1 was transformed into the Agrobacterium strain GV3101, which was mixed with a pTRV1-transformed strain and then delivered into leaves of $N$. benthamiana plants by agroinfiltration with a needle-free syringe to initiate gene silencing. The VIGS analyses were repeated twice. For each experiment, 10 plants were analyzed.

Transient RNA interference assay. The full-length open reading frame (ORF) of $N b U E P 1$, amplified using the primer pair NbUbi-F/NbRlp-R, was inserted in reverse and forward directions into the two sides of a bean nitrate reductase gene intron with the sites $X h o \mathrm{I} / K p n \mathrm{I}$ and $X b a \mathrm{I} /$ BamHI, respectively, in the pCAMBIA1301-based RNA interference (RNAi) vector (pCRNAi$N b U E P 1$ ) so that an inverted repeat (IR) sequence of $N b U E P 1$ could be formed to initiate efficient RNAi. The recombinant plasmid pCRNAi-NbUEPl was transformed into the Agrobacterium strain GV3101. The bacterial suspension, with an optical density at $600 \mathrm{~nm}\left(\mathrm{OD}_{600}\right)$ value of 2.0 was infiltrated into leaves of $N$. benthamiana and $N$. tabacum plants at the six-leaf stage and flowering stage, respectively. The transient RNAi assays were repeated twice. For each experiment, eight plants were analyzed.

Transient overexpression assay. The full-length ORF of NbUEP1 amplified using the primer pair NbUbi-F/NbRlp-R was inserted into the overexpression vector pCHF3 after the CaMV $35 \mathrm{~S}$ promoter by BamHI/SalI. The recombinant plasmid pCHF3NbUEP 1 was transformed into the Agrobacterium strain GV3101. The viral gene-silencing suppressor P19 was coexpressed to avoid possible silencing of $N b U E P 1$ in the transient overexpression assay. The mixed Agrobacterium suspension with a final $\mathrm{OD}_{600}$ value of 2.0 for both $N b U E P 1$ - and $P 19$-expressing bacteria was infiltrated into leaves of $N$. benthamiana plants at the six-leaf stage. The transient overexpression assays were repeated twice. For each experiment, six to eight plants were analyzed.

Construction of stable UEP1-RNAi plants. Stable UEP1RNAi tobacco plants were constructed by Agrobacterium-mediated transformation of pCRNAi-NbUEP1 into leaf discs of $N$. tabacum cultivar K326 following previously described procedures (Cai et al. 2007). The $T_{2}$ homozygous lines with single-copy insertion were selected for further functional analyses. The RT-quantitative PCR (RT-qPCR) assay was performed using primers NbUEP-F and NbUEP-R to detect the expression level of the UEP1 gene in each line, as described in more detail below.

Gene expression analyses. Reverse-transcription PCR and RT-qPCR analyses and consequent statistical data analyses were conducted as described (Cao et al. 2016; Zhao et al. 2013). RTqPCR data were statistically analyzed using SPSS software (version 19.0; IBM). Significant difference between the mean values of three independent experiments was determined with Duncan's multiple range test $(P<0.05)$ or $t$ test $(P<0.01)$. The data represent means \pm standard error (SE).

Trypan blue staining assay. Leaf discs were depigmentized in an ethanol/chloroform mixture $(3: 1[\mathrm{vol} / \mathrm{vol}])$ for $4 \mathrm{~h}$, and then stained in staining solution ( $10 \mathrm{ml}$ of lactic acid, $10 \mathrm{ml}$ of glycerol, and $10 \mathrm{ml}$ of trypan blue solution at $1 \mathrm{mg} / \mathrm{ml}$ ) for $4 \mathrm{~h}$, followed by destaining in saturated chloral hydrate for $2 \mathrm{~h}$. The destained leaf discs were mounted on slides in $70 \%$ glycerol for microscopy observation. The trypan blue staining assays were conducted for $U E P 1$-RNAi tobacco lines 2, 4, and 5 and repeated twice. For each experiment, six plants were analyzed.

Plant inoculation and resistance evaluation. Resistance of UEP1-RNAi tobacco plants to oomycete Pythium aphanidermatum, viruses Tobacco mosaic virus (TMV) and Cucumber mosaic virus (CMV), and nematode Meloidogyne incognita was examined through inoculation analyses. For $P$. aphanidermatum, leaves were inoculation with mycelial plugs and disease lesion diameter was measured as described (Zhao et al. 2013). For TMV and CMV, virus was extracted from diseased $N$. tabacum K326 and N. glutinosa leaves, respectively, displaying strong viral symptoms $(10 \mathrm{ml}$ of phosphate-buffered saline [PBS] per $1 \mathrm{~g}$ of leaves). Two leaves per plant were dusted with carborundum and inoculated by gently rubbing with the viral crude extracts ( $200 \mu \mathrm{l} / \mathrm{leaf}$, PBS as control). Five minutes later, the inoculated leaves were rinsed with water to remove the dusts. The inoculated plants were grown at $25^{\circ} \mathrm{C}$ with a photoperiod of $16 \mathrm{~h}$ of light and $8 \mathrm{~h}$ of darkness. The cDNA sequences derived from coat protein and movement protein genes were detected by RT-qPCR using gene-specific primers. For $M$. incognita, each plant was inoculated with $5 \mathrm{ml}$ of secondstage juvenile (J2) nematode inocula containing approximately 2,000 nematodes. The inocula were poured into a hole that was punched 8 to $10 \mathrm{~cm}$ into soils at approximately $2 \mathrm{~cm}$ from plant stem. The inoculated plants were grown at $25^{\circ} \mathrm{C}$ with a photoperiod of $16 \mathrm{~h}$ of light and $8 \mathrm{~h}$ of darkness. Root-knot formation of inoculated plants was investigated following a described 0-to-5 galling index system (Barker 1985). Disease index was calculated as $100 \times \Sigma(N \times R) /(8 \times 5)$, where $N=$ number of plants in each rate, $R=$ rate value, $8=$ number of total plants, and $5=$ the highest rate value

The inoculation analyses were conducted three times in plants of UEP1-RNAi tobacco lines 2, 4, and 5 with similar results. Eight plants from each line were used for each inoculation experiment. The inoculation data were statistically analyzed using SPSS software (version 19.0; IBM). A significant difference between 
the mean values of three independent experiments was determined with the $t$ test $(P<0.01)$. The data represent means \pm SE.

Microarray analysis for transcript profiling. The necrotic tissues of the lower leaves of UEPI-RNAi line 4 tobacco plants and the healthy tissues of the corresponding leaves of non-RNAi control plants were sampled. Total RNA was extracted using Trizol and purified using a NucleoSpin RNA clean-up kit, which was then subjected to labeling using the JingXin cRNA amplification label kit. Briefly, the first-strand cDNA was synthesized from purified total RNA using CbcScript enzyme and T7 Oligo (dT) primer. RNA in the generated product was cleaved by RNase $\mathrm{H}$, and the secondstrand cDNA was synthesized from the released short RNA fragments using DNA polymerase. The purified double-stranded cDNA was transcribed into cRNA by T7 Enzyme Mix. The purified cRNA was reverse transcribed into DNA by CbcScript II enzyme and Random primer. The DNA was labeled with fluorescent dNTP during synthesis of the second-strand DNA by Klenow Fragment enzyme and Random primer. The labeled DNA was hybridized with Agilent Tobacco mRNA 4-by-44k microarray chip. The hybridization signal was obtained by scanning the chip with an Agilent G2565CA Microarray Scanner, and analyzed by Feature Extraction software. The original data were further analyzed by GeneSpring GX software and normalized using a percentile shift approach. The fold change of gene expression in UEP1-RNAi plants relative to non-RNAi control plants was calculated. To solve the problem of lacking annotation of tobacco genes, agriGO software tools (gene ontology [GO] analysis toolkit and database for agricultural community, http://bioinfo.cau.edu.cn/agriGO/) were used to functionally annotate the genes under the setup of false discovery rate $<$ 0.05 (Du et al. 2010). The upregulated and downregulated genes associated with each statistically significant $(P<0.05, t$ test $) \mathrm{GO}$ term were counted and analyzed respectively.

\section{RESULTS}

Cloning and sequence analyses of a Nicotiana benthamiana $\boldsymbol{U E P}$ gene $N \boldsymbol{B U E P 1}$. Searching against the $N$. benthamiana expressed sequence tag database retrieved a set of Ub-related sequences, among which was a full-length cDNA sequence encoding for a UEP protein (AY912494). Using the primers designed in accordance with this $U E P$ gene, a $U E P$ cDNA sequence was amplified and cloned from $N$. benthamiana. Sequencing results demonstrated that it is a full-length $U E P$ gene of $471 \mathrm{bp}$ in size, encoding for a fusion protein of a Ub (76 aa) at its $\mathrm{N}$ terminus to a s27a-type ribosomal protein ( $80 \mathrm{aa})$ at its $\mathrm{C}$ terminus and, thus, was provisionally named NbUEP1 (National Center for Biotechnology Information accession number MG333694) (Fig. 1A). BLASTn searching analysis indicated that the $N b U E P 1$ nucleotide sequence was most similar to an N. tabacum UEP gene (AJ223329), with an identity of $96 \%$, followed by to the $N$. benthamiana UEP gene (AY912494), with an identity of 91\% (Supplementary Fig. S1).

VIGS of NbUEP1 caused strong necrosis. Taking advantage of $N$. benthamiana as a model VIGS plant species, we preformed VIGS analysis to understand the function of $N b U E P 1$. To ensure that effects from both $\mathrm{Ub}$ and ribosomal protein moieties could be observed, a 383-bp sequence of NbUEP1, including both $\mathrm{Ub}$ and ribosomal protein moieties, were cloned into TRV-based VIGS vector for silencing analyses. Remarkably, necrosis developed in the agroinfiltrated areas at 3 to 4 days postinfiltration (dpi) and spread beyond the infiltrated areas to cause the whole infiltrated leaves to wilt at $5 \mathrm{dpi}$. Later, the necrosis expanded beyond the infiltrated leaves to the stem and up uninfiltrated leaves and, ultimately, the whole plant withered at 7 to 8 dpi (Fig. 1B). Transcript accumulation analysis by RT-qPCR showed that the NbUEPl gene had been efficiently silenced (Fig. 1B). This result indicated that silencing of NbUEP1 led to intensive cell death in plants.

Transient RNAi but not overexpression of NbUEP1 resulted in strong cell death. To confirm the VIGS result and avoid the effect from TRV virus, another gene-silencing strategy RNAi was employed to analyze the function of NbUEPI in cell death. The full-length ORF of $N b U E P 1$ was inserted in reverse and forward directions into the two sides of an intron, respectively, in the pCAMBIA1301-based RNAi vector (pCRNAi-NbUEPl) so that an IR sequence of $N b U E P 1$ could be formed to initiate efficient RNAi (Fig. 2A). Agrobacterium strain GV3101 transformed with pCRNAi-NbUEP1 was infiltrated into leaves of $N$. benthamiana plants. Cell death occurred strongly in the infiltrated areas and the time required for complete necrosis development depended on the stage of plant for infiltration. It took 4 days for leaves of six-leaf stage plants whereas it needed 6 days for those of flowering stage plants (Fig. 2B and C). In addition, infiltration of the same Agrobacterium in N. tabacum also caused necrosis as observed in N. benthamiana (Fig. 2D). Transcript detection by RT-qPCR showed that the UEPl gene in both plant species had been efficiently silenced (Fig. 2E). It is not unexpected considering that NbUEPI is $96 \%$ identical to an N. tabacum UEP gene (AJ223329).

A 1 76 156

B

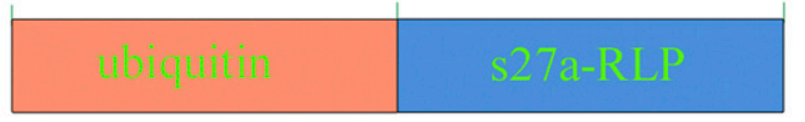

\section{NbUEP1}
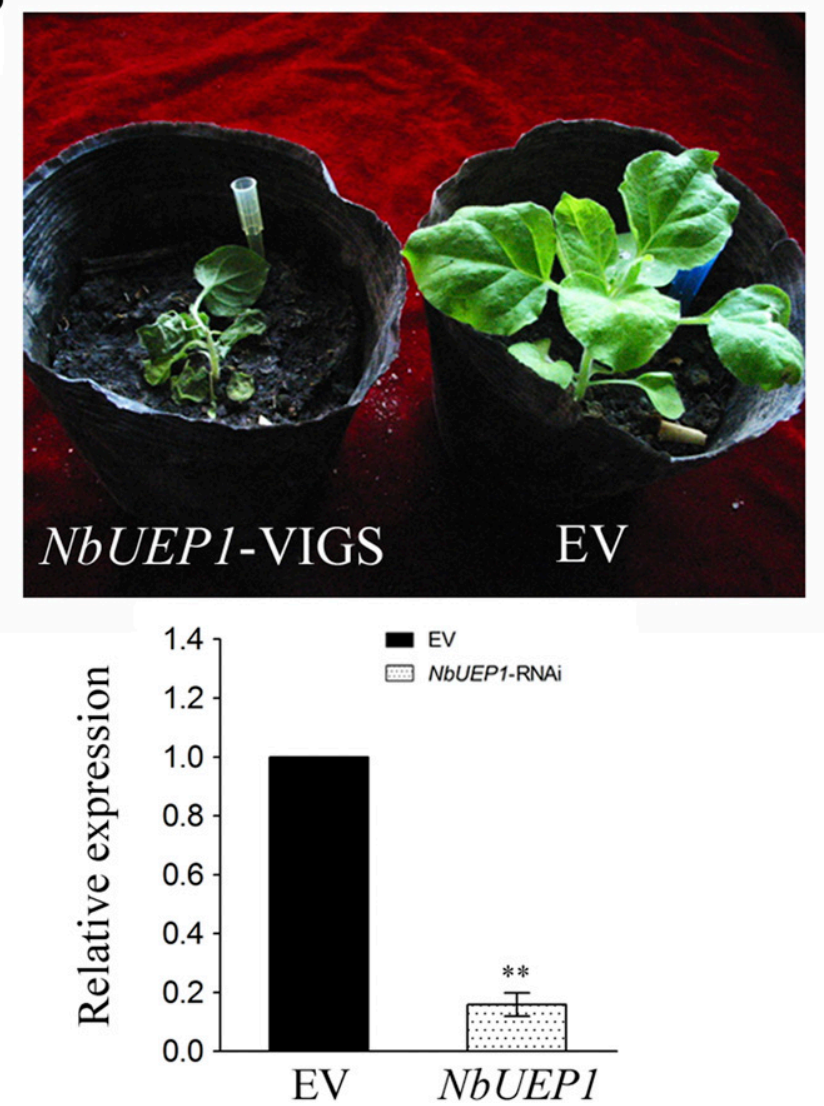

Fig. 1. Virus-induced gene silencing (VIGS) of NbUEP1 resulted in intensive necrosis. A, Structure of protein sequence predicted from NbUEP1 cloned in this study. B, Symptoms of Tobacco rattle virus-induced gene silencing of NbUEP1 in Nicotiana benthamiana at 7 days postagroinfiltration (up) and efficiency of $N b U E P 1$ gene silencing detected by quantitative real-time polymerase chain reaction (down). $18 \mathrm{~S}$ ribosomal DNA serves as an inner control. Data represent mean \pm standard error of three independent experiments. Double asterisks (**) indicate a significant difference of NbUEP1 transcript accumulation between $U E P 1$-silenced plants and empty vector (EV) control plants $(P<0.01, t$ test $)$. 
Additionally, the RNAi enhanced the expression of HIN1, a cell death-related gene (Fig. 2F), confirming in the molecular level that RNAi of $N b U E P 1$ resulted in cell death.

To further understand the role of $N b U E P 1$ in cell death, a transient overexpression assay was carried out for this gene. Agroinfiltrated areas already showed enhanced expression of NbUEP 1 at 3 dpi, with Agrobacterium expressing NbUEP1 under CaMV 35S promoter, but did not exhibit visible necrosis even at 6 dpi (Supplementary Fig. S2).

Collectively, these results reveal that RNAi but not overexpression of NbUEP1 induces strong cell death in both $N$. benthamiana and N. tabacum.

Stable NbUEP1-RNAi plants constitutively developed cell death and defense responses. To confirm the transient RNAi assay result and further understand the role of $N b U E P 1$ in disease resistance of common tobacco, stable $N b U E P 1-\mathrm{RNAi}$ plants were constructed by transformation of pCRNAi-NbUEP1 into $N$. tabacum. Six independent lines were generated. Genetic segregation analyses for $\mathrm{T} 1$ seed showed that lines 2,4 , and 5 contained a single-copy insertion of NbUEPl; thus, their $\mathrm{T}_{2}$ homozygous lines were selected for further functional analyses. The RT-qPCR assay demonstrated that transcript of $U E P 1$ in lines 2 , 4 , and 5 was only $25.5,17.5$, and $24.3 \%$, respectively, of that in control plants (Fig. 3A), demonstrating that the UEP1 gene had been efficiently knocked down in these RNAi lines.

$U E P 1-R N A i$ plants showed some specific phenotypes compared with non-RNAi control plants. In UEP1-RNAi plants, the lower leaves narrowed and crinkled and formed water-soaked necrotic spots, which cohered at a later stage, while some leaves were mosaic (Fig. 3B and C). Nevertheless, severe phenotypes only exhibited in the lower leaves, and these plants could grow up and fruit normally.

\section{A}
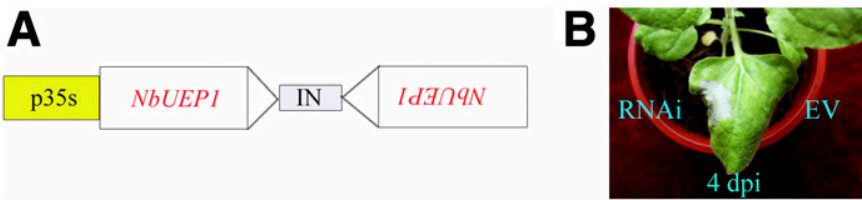

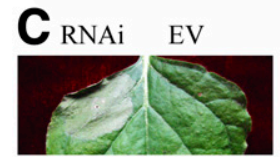

$6 \mathrm{dpi}$

E

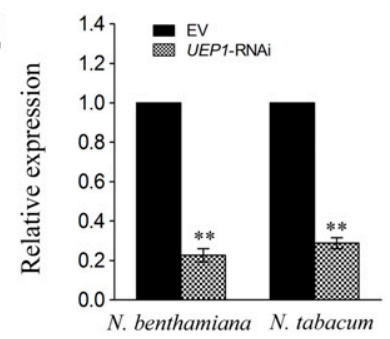

9 dpi
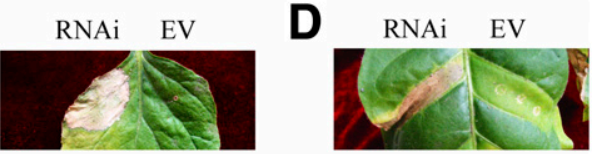

9 dpi

E

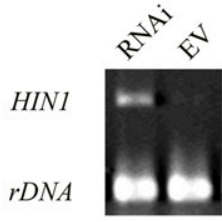

Fig. 2. Transient RNA interference (RNAi) of NbUEPl caused strong cell death. A, Diagram for structure of NbUEPl-IR sequence inserted in RNAi vector. Symptoms of transient NbUEP1-RNAi (RNAi) in B, Nicotiana benthamiana at the six-leaf stage and $\mathbf{C}$, flowering stage. $\mathrm{EV}=$ infiltration with Agrobacterium transformed with empty vector. D, Symptoms of transient NbUEP1-RNAi in N. tabacum at the six-leaf stage. Efficiency of E, NbUEP1RNAi and F, HIN1 expression in transient $N b U E P 1-R N A i$ leaves of $N$. benthamiana plants at 3 days postinfiltration (dpi). Efficiency of NbUEP1-RNAi was analyzed by quantitative real-time polymerase chain reaction (RT-qPCR). HIN1 expression was detected by RT-PCR. Polyacrylamide gel electrophoresis profiles of RT-PCR products were shown. 18S ribosomal DNA served as an inner control for the loading check.
To further understand the cell death that appeared in UEPlRNAi plants, a trypan blue staining assay was performed and expression of cell death-related gene HIN1 and pathogenesisrelated $(P R)$ genes were monitored. A trypan blue staining assay showed that the necrotic tissues of UEP1-RNAi plants were clearly stained into blue, whereas no-symptom and mosaic tissues of UEP1-RNAi plants were not, as observed for those of nonRNAi control plants (Fig. 3D). This result confirmed that cell death, indeed, occurred in the necrotic tissues of UEPI-RNAi plants. Moreover, the RT-qPCR assay revealed that, compared with the non-RNAi control plants, expression of two $P R$ genes ( $P R 1$ and $P R 5$ ), and the cell death-related gene HIN1 was strongly upregulated by 19.1-fold, 26.2-fold, and 2.7-fold, respectively, in the necrotic tissues of UEP1-RNAi plants, whereas expression of these genes in the other two types of tissues of UEP1-RNAi plants was only slightly increased (Fig. 3E).

Taken together, our results obtained using UEP1-RNAi plants reveal that RNAi of $U E P 1$ constitutively activates strong cell death and defense responses in tobacco plants.

$U E P 1$ widely influences the resistance against various pathogens. To probe the role of $U E P I$ in disease resistance of common tobacco, inoculation analyses were conducted in stable UEP1-RNAi tobacco plants against a variety of pathogens, including oomycete $P$. aphanidermatum, viruses TMV and CMV, and nematode $M$. incognita.

RNAi of UEPI enhanced resistance to the oomycete pathogen $P$. aphanidermatum. The fully expanded symptomless leaves of UEP1-RNAi plants were inoculated with the pathogen mycelial plugs. Inoculation experiments demonstrated that, after inoculation, disease necrosis developed much slower in UEP1-RNAi plants than non-RNAi control plants. At $27 \mathrm{~h}$ postinfiltration, the lesion was only $2.0 \mathrm{~cm}$ in diameter in UEP1-RNAi plants whereas that had expanded to $2.8 \mathrm{~cm}$ in diameter in non-RNAi control plants (Fig. 4). This result suggests that UEPl-RNAi plants display increased resistance to $P$. aphanidermatum.

RNAi of $U E P 1$ augmented resistance to the viral pathogens TMV and CMV. The symptomless leaves of UEPI-RNAi plants were rubbing inoculated with the viral extracts. TMV inoculation experiments showed that, after inoculation, viral disease developed similarly in UEP1-RNAi plants and non-RNAi control plants. Growth and flowering were suppressed in both types of plants (Fig. 5A). However, the RT-qPCR assay showed that, in UEPI-RNAi plants, the cDNA sequences derived from TMV coat protein $(\mathrm{CP})$ and movement protein (MP) genes only accumulated to less than half of those in non-RNAi control plants (Fig. 5B), indicating that UEPI-RNAi plants induced resistance to TMV. Additionally, CMV inoculation analysis showed that growth and development of inoculated non-RNAi control plants were severely repressed. These plants could not flower. However, growth and development of inoculated UEP1-RNAi plants were much less suppressed. They could grow up and flower (Fig. 5C). Furthermore, the RT-qPCR assay demonstrated that, in UEPl-RNAi plants, the cDNA sequences derived from CMV CP and MP genes only accumulated to approximately $30 \%$ of those in non-RNAi control plants (Fig. $5 \mathrm{D})$, indicating that UEPI-RNAi plants enhanced resistance to CMV.

RNAi of $U E P 1$ reduced resistance to the nematode pathogen $M$. incognita. Roots of UEPI-RNAi plants were drench inoculated with the $\mathrm{J} 2$ nematodes. At 30 days after nematode inoculation, aboveground parts of UEPI-RNAi plants and nonRNAi control plants seemed to be similar. However, the roots of UEP1-RNAi plants formed many more root-knots than those of non-RNAi control plants. Approximately $80 \%$ of the roots of UEP1-RNAi plants formed root-knots whereas this percentage was only approximately $25 \%$ for non-RNAi control plants. Meanwhile, the number of root-knots per root was also much higher in UEPl-RNAi plants than in non-RNAi control plants (Fig. 6A). Moreover, a disease index, calculated based on a 0 -to-5 
galling index system, was significantly higher for UEP1-RNAi plants (89.2) than non-RNAi control plants (37.5) (Fig. 6B). This result indicates that UEP1-RNAi plants exhibit decreased resistance to $M$. incognita.
Collectively, our data provided evidence that UEP1 is broadly involved in tobacco disease resistance against a variety of pathogens. This gene negatively affects resistance to oomycete pathogen $P$. aphanidermatum and viral pathogens TMV and CMV,

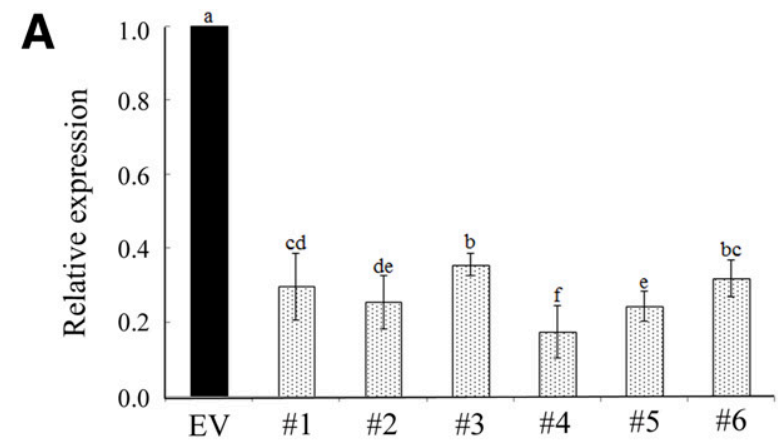

B

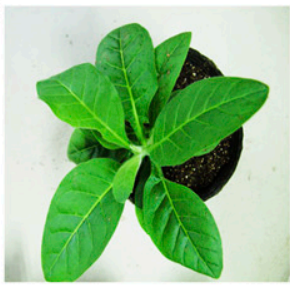

$\mathrm{EV}$

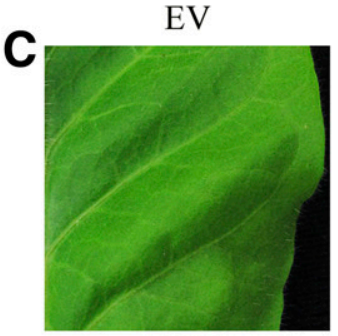

D

EV

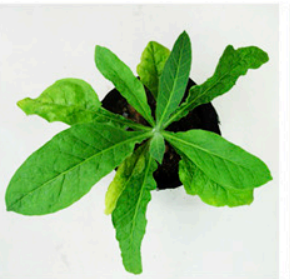

\#2

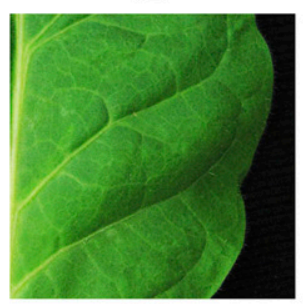

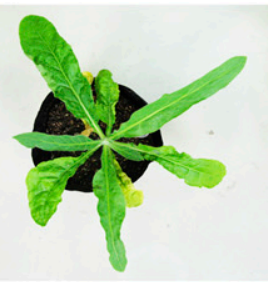

\#4

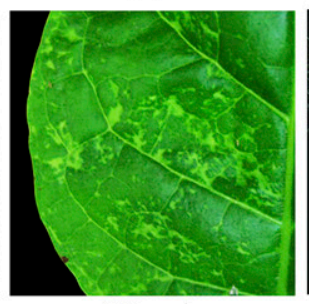

Mosaic

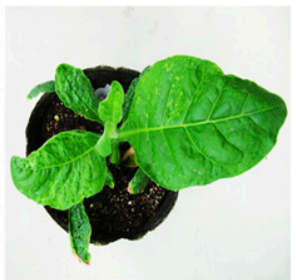

\#5

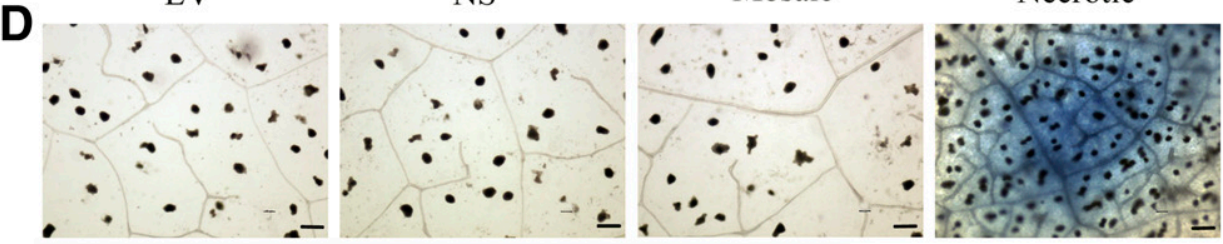

E

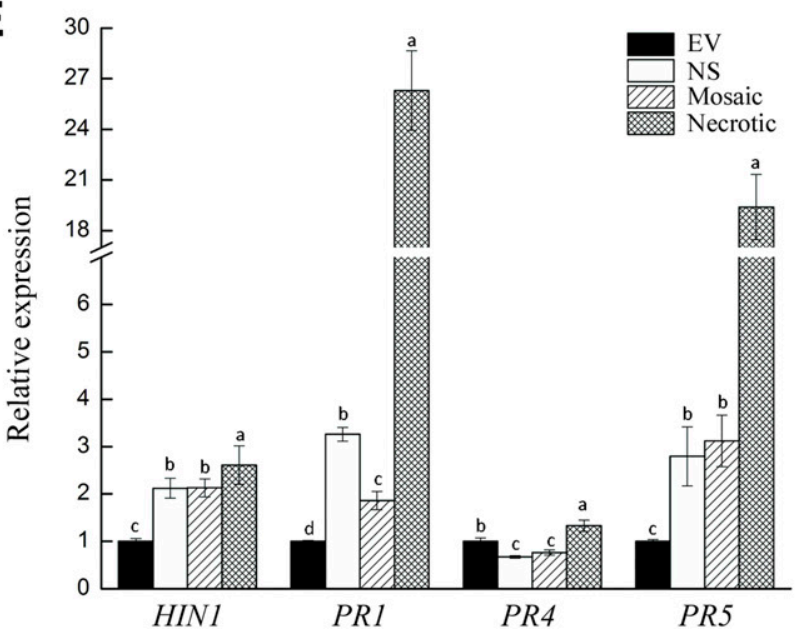

Fig. 3. Stable NbUEP1-RNA interference (RNAi) plants constitutively developed cell death and defense responses. A, Expression level of $U E P 1$ in stable RNAi tobacco lines relative to empty-vector (EV) control plants. Quantitative real-time polymerase chain reaction (RT-qPCR) results are shown. Data represent mean \pm standard error of three independent experiments. Small letters indicate significant difference $(P<0.05$, Duncan's multiple range test). B, Typical plants of RNAi tobacco. C, Magnified view for various symptoms developed in lower leaves of UEPI-RNAi plants. Both single and cohered necrotic spots were shown. NS = no symptoms. D, Light microscopy observations for trypan blue-stained tissues showing typical symptoms in line 4 . Bar $=100 \mu \mathrm{M}$. E, Expression of hypersensitive response marker and pathogenesis-related (PR) genes in UEP1-RNAi plants of line 4. Data were analyzed and shown as in A. 
whereas it positively modulates resistance to nematode pathogen M. incognita.

Transcript profiling for $U E P 1$-regulated resistance. Given that Ub not only functions by protein degradation but also plays important roles in transcriptional regulation (Conaway et al. 2002), to explore the molecular mechanisms underlying UEP1modulated tobacco disease resistance, comparative transcript profiling between necrotic tissues of UEP1-RNAi plants and healthy tissues of non-RNAi control plants were analyzed using the Agilent Tobacco mRNA 4-by-44k microarray. Genes differentially expressed (DE) between the two types of plants were selected according to the following criteria: (i) absolute fold change $\geq 2.0$, (ii) flag signal for both samples is detectable, and (iii) processed signal value for both samples $\geq 800$. In total, $915 \mathrm{DE}$ genes were identified based on the above criteria, among which 400 were upregulated while 515 were downregulated in UEP1-RNAi plants compared with non-RNAi control plants (Supplementary Table S2). To solve the problem of lacking annotation of tobacco genes, agriGO software tools were used to functionally annotate the DE genes (Du et al. 2010). Consequently, 439 (48\%) DE genes were successfully annotated. Among the statistically significant $(P<$ $0.05, t$ test) GO terms, 13 of Biological Process, 10 of Molecular Function, and 9 of Cellular Component terms were enriched (comprising at least five genes) (Fig. 7). For the Biological Process class, DE genes associated with eight terms (electron transport chain, oxidation-reduction, nucleosome assembly, thiamine biosynthetic process, thiazole biosynthetic process, proteolysis, intracellular signal transduction, and starch biosynthetic process) were dominantly downregulated (at least five more than upregulated genes and described as "enriched" hereafter), which was the opposite of those associated with the terms calcium-mediated signaling and cellular glucan metabolic process (Fig. 7A). Regarding the Molecular Function class, downregulated DE genes were enriched for seven terms (metal ion binding, electron carrier activity, two iron-two sulfur cluster binding, copper ion binding, protein heterodimerization activity, oxidoreductase activity, and phosphatidylinositol phospholipase $\mathrm{C}$ activity), whereas upregulated DE genes were enriched for only one term (xyloglucanxyloglucosyl transferase activity) (Fig. 7B). For the Cellular Component class, downregulated DE genes were enriched for intracellular localization-associated terms (chloroplast, chloroplast thylakoid membrane, chloroplast stroma, nucleosome, amyloplast, and intracellular part), while upregulated DE genes were enriched for extracellular localization-associated terms (cell wall and extracellular region) (Fig. 7C). To verify the expression patterns of the DE genes, expression of eight DE genes, which are potentially associated with cell death or defense, was monitored in UEPIRNAi plants and non-RNAi control plants, respectively, by RTqPCR analysis. Results obtained from this RT-qPCR analysis were similar to those from the microarray transcript profiling assay (Fig. 8). Together, these results indicate that intracellular activities such as photosynthesis are strongly repressed while extracellular activities, including cell wall modification, are significantly promoted in the necrotic tissues of UEP1-RNAi plants compared with the healthy tissues of non-RNAi control plants.

To further find out the genes probably involved in UEP1mediated resistance, the DE genes with an absolute fold change $\geq 4$ and those encoding a known resistance-related protein were selected (Table 1). Among the most upregulated DE genes were those encoding for a set of glycine-rich proteins (GRPs) (approximately +4.9- to 21.6-fold), Cf-9/Avr9 rapidly elicited protein 44 (ACRE44)/linoleate 13S-lipoxygenase 3-1 (+12.6-fold), several jasmonate-ZIM-domain (JAZ) proteins (approximately +5.0- to 11.4-fold), a GRAS2 transcription factor (+8.6-fold), and a leucine-rich repeat (LRR) family protein (+8.4-fold) (Table 1), indicating that the JAZ-dependent jasmonate (JA) pathway, ACRE44-dependent lipid pathway, GRAS2, LRR family protein, and GRPs might be associated with UEPl-mediated resistance. In addition, DE genes encoding for $\mathrm{Ca}^{2+}$-signaling components, including CaMs, CPKs, and CIPKs; a set of WRKY transcription factors, including WRKY6, -31, -33, and -40; and redox regulators, including peroxidase protein PER12 and thioredoxin H-type protein $\mathrm{TH} 9$, were also significantly upregulated (Table 1), suggesting that $\mathrm{Ca}^{2+}$ signaling, WRKY transcription factors, and redox modulation might also be related to UEPl-mediated resistance.

Additionally, in contrast to the above DE genes, the following were strongly downregulated in UEP1-RNAi plants: a copper amine oxidase $(\mathrm{CuAO},-11.3$-fold $)$, three vacuolar processing enzymes (VPEs) (approximately -6.7- to -7.5-fold), RD21 (-4.3fold), phospholipase A2 ( -4.5 -fold), glycerate dehydrogenases (-4.2-fold), a phosphoinositide binding protein (-4.2-fold), a set of EFEs/ACOs (approximately -2.0- to -3.3-fold), and two ATG8s (approximately -2.0 - to -2.5 -fold) (Table 1 ). These data demonstrate that the $\mathrm{CuAO}-$ mediated nitrous oxide $(\mathrm{NO})$ and reactive oxygen species (ROS) production; VPE- and RD21-mediated cell
A

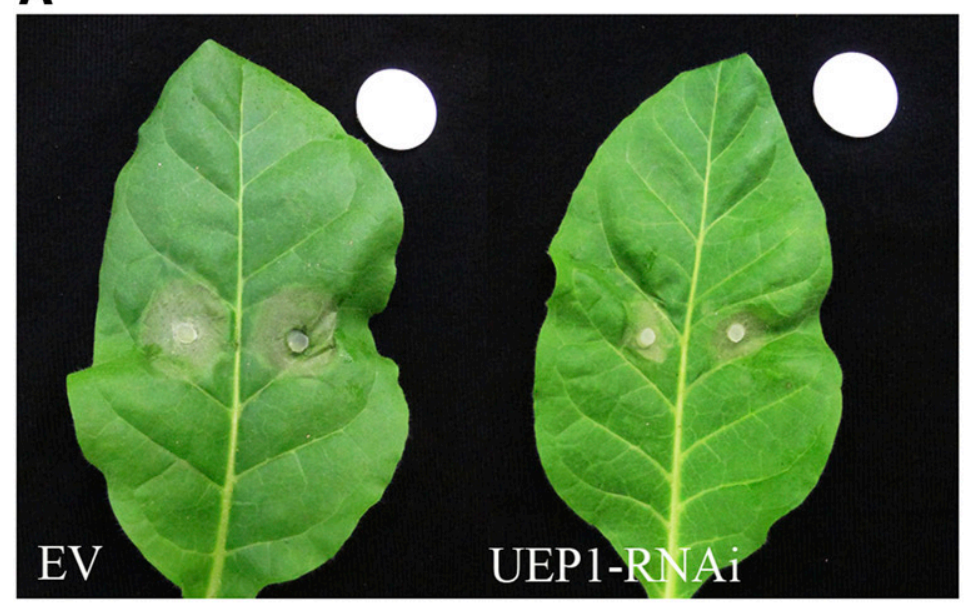

B

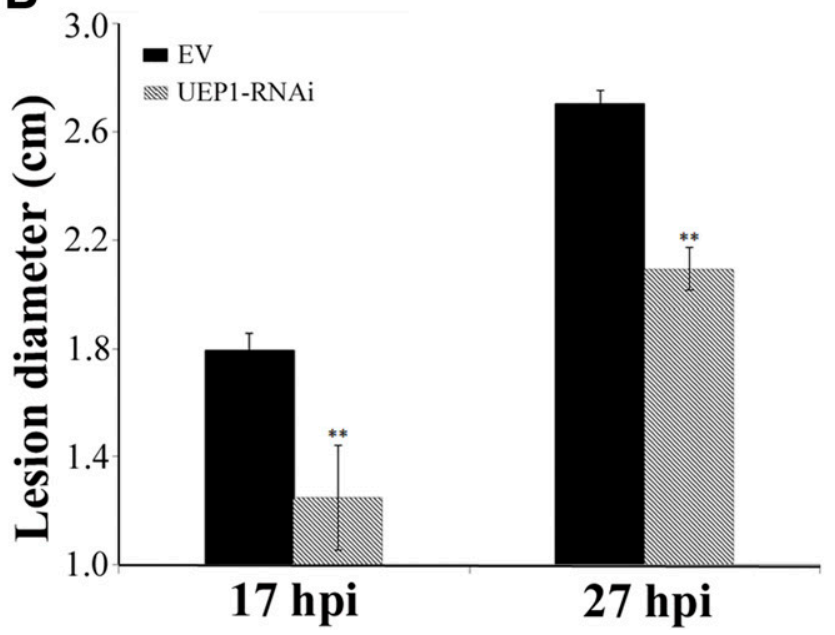

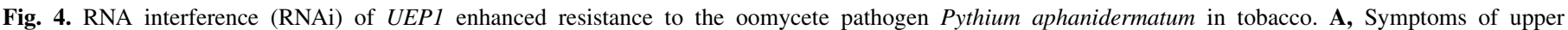

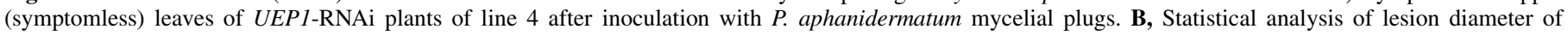

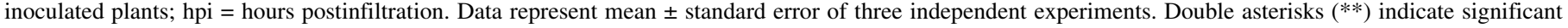
difference of lesion diameter between $U E P 1$-RNAi plants and empty-vector (EV) control plants $(P<0.01, t$ test). 
death and disease resistance; phospholipase A2, glycerate dehydrogenases, and the phosphoinositide binding protein-mediated lipid signaling; EFE/ACO-dependent ethylene (ET) biosynthesis; and ATG8-mediated autophagy, cell death, and resistance might be linked to $U E P 1$-mediated resistance.

Interestingly, a set of Ub genes such as UBQ3 and UBQ10 were significantly downregulated by approximately 2.1 - to 2.7 -fold (Table 1), implying that Ub genes might be cosuppressed with the UEP1 gene in UEP1-RNAi plants due to their high homology in sequence. Considering the molecular relationship between UEP and $\mathrm{Ub}$, this provided another layer of evidence for the efficiency of $U E P 1$ gene silencing in UEP1-RNAi plants, and implied that
Ub-26S proteasome system-mediated protein modification is involved in regulating $U E P 1$-mediated resistance.

\section{DISCUSSION}

The pivotal role of $\mathrm{Ub}$ as protein modifier in various biological processes has been well recognized in all eukaryotes. Nevertheless, the function of Ub fusion protein UEP in plants is rarely studied. In particular, direct evidence for the role of plant UEPs in plant defense against pathogens is still unavailable. In this study, we demonstrated that a tobacco Ub-S27a-type UEP gene, NbUEP1, regulates plant cell death and influences disease resistance against a variety of
A

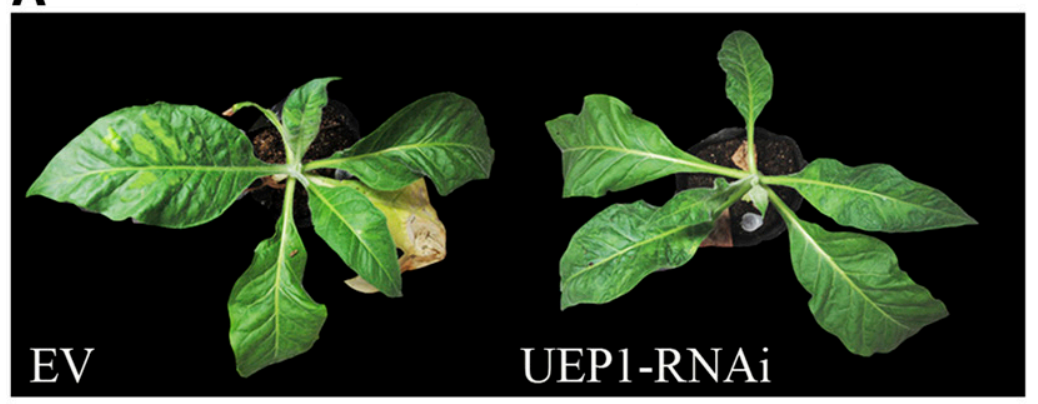

C
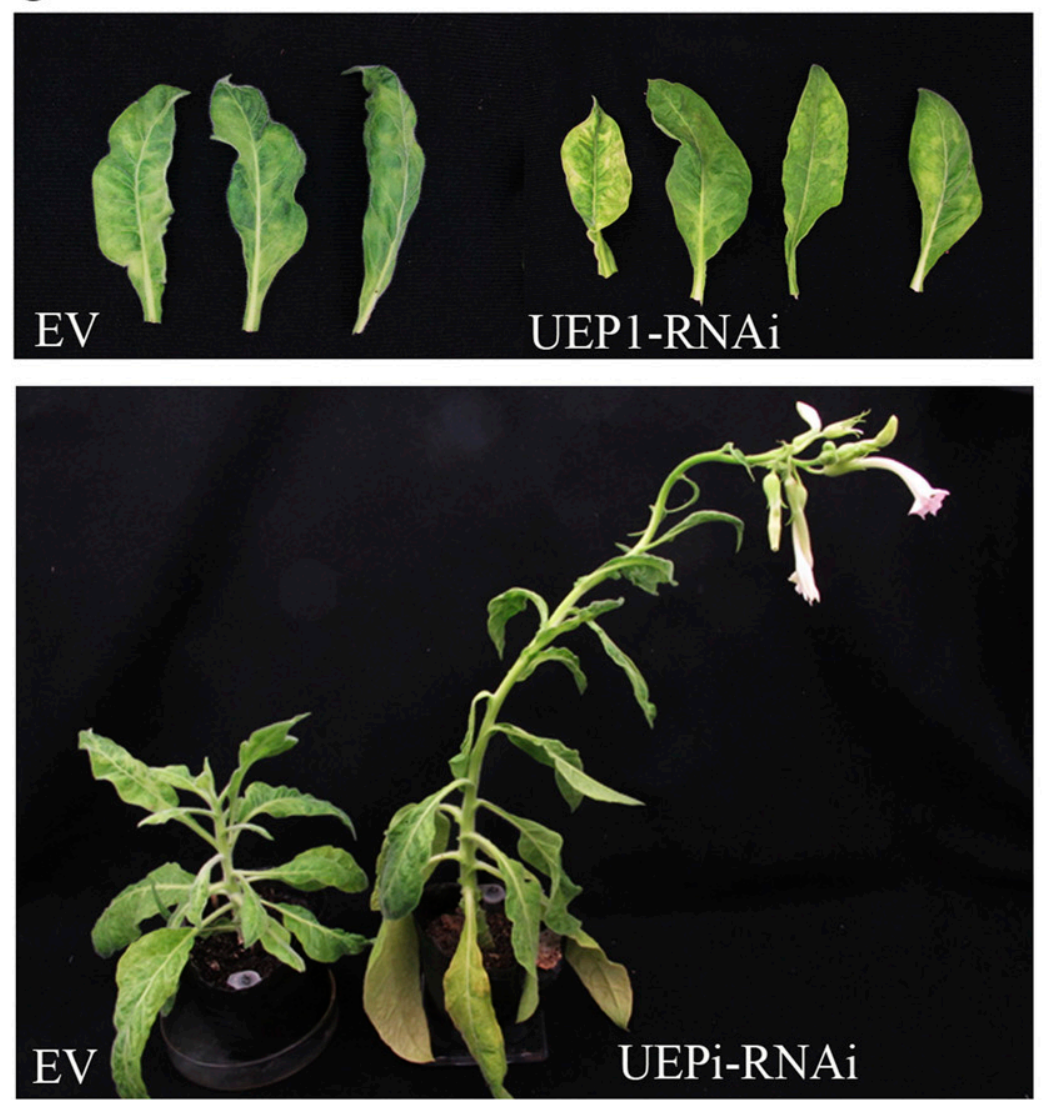

B

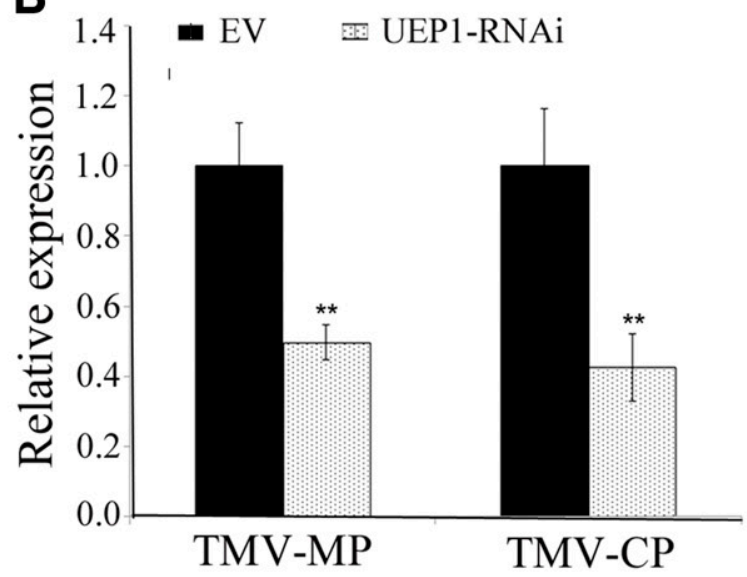

D

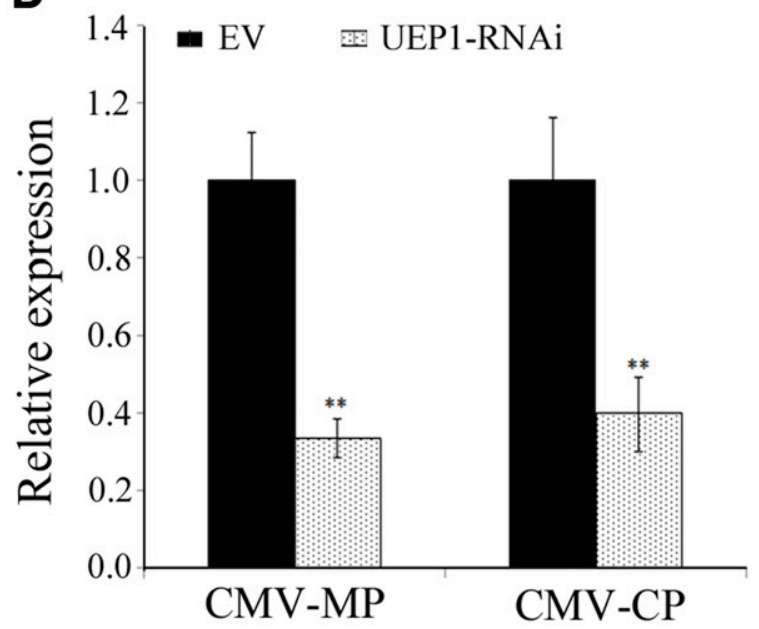

Fig. 5. RNA interference (RNAi) of UEP1 augmented resistance to the viral pathogens Tobacco mosaic virus (TMV) and Cucumber mosaic virus (CMV) in tobacco. A, Symptoms of UEP1-RNAi plants of line 4 after rubbing inoculation of upper (symptomless) leaves with TMV. EV = empty vector control. B, Expression of TMV movement protein (MP) and coat protein (CP) genes in inoculated plants. Quantitative real-time polymerase chain reaction (RT-qPCR) results are shown. Data represent mean \pm standard error of three independent experiments. Double asterisks $(* *)$ indicate significant difference of expression level between UEP1-RNAi plants and EV control plants $(P<0.01, t$ test). C, Symptoms of $U E P 1$-RNAi plants of line 4 after rubbing inoculation of upper (symptomless) leaves with CMV. D, Expression of CMV MP and CP genes in inoculated plants. RT-qPCR results are shown. Data were analyzed and shown as in B. 
pathogens, potentially through modulating complex pathways, including JA and ET pathways; autophagy; and CuAO-, VPE-, and RD21-mediated pathways. Our results provided evidence for the contribution of plant UEP to modulating cell death and disease resistance.

Tobacco $U E P 1$ strongly regulates cell death. Three layers of evidence obtained in this study reveal the important role of tobacco UEP1 in regulating cell death. First, silencing of NbUEP1 through VIGS analysis in $N$. benthamiana led to rapid and strong systemic cell death and whole-seedling wilt within a week (Fig. 1). Second, to rule out the interference of viruses in VIGS assays, we conducted RNAi analyses. Transient RNAi of NbUEPl induces strong cell death in the agroinfiltrated areas of both $N$. benthamiana and N. tabacum plants (Fig. 2). Third, stable UEP1-RNAi plants were constructed and these plants constitutively developed cell death. To characterize the cell death, in addition to macroscopic observation, we performed trypan blue staining assays and celldeath-related and defense-related gene expression analysis. Results showed that the necrotic areas were deeply stained by trypan blue, and cell-death-related and defense-related genes were strongly expressed in the necrotic tissues (Fig. 3). Nevertheless, whether the cell death formed in the UEPl-RNAi plants is a hypersensitive response (HR) requires further verification by other analyses such as the TUNEL assay. It has been reported that tobacco plants transformed with a wild-type Ub gene showed less Ub accumulation due to cosuppression of the endogenous Ub gene, and these plants displayed strong necrosis (Becker et al. 1993). A similar phenomenon was also observed in a transient in planta expression assay for HR-eliciting gene screening (Karrer et al. 1998). In addition, tobacco plants transformed with a variant $\mathrm{Ub}$ gene (Ub R48) in which lys 48 is replaced by arg, acting as a specific inhibitor of Ub-dependent proteolysis, contained normal levels of free Ub but were deficient in Ub-dependent proteolysis. These plants not only exhibited necrotic lesions but also induced expression of $P R-1$ gene and enhanced resistance to TMV (Becker et al. 1993). In addition, the Ub R48-transgenic plants showed leaf curling symptoms

\section{A}
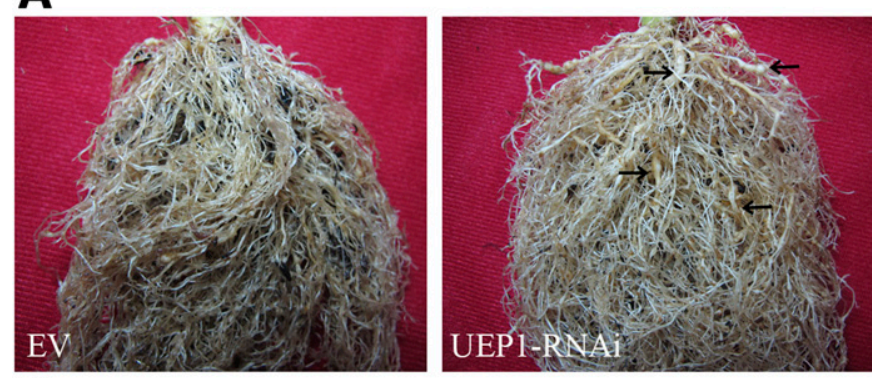

B

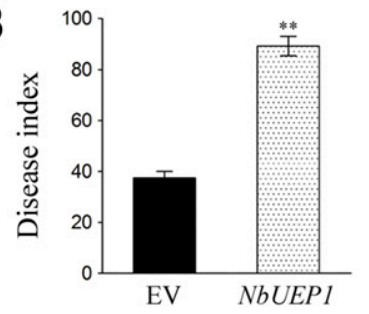

Fig. 6. RNA interference (RNAi) of $U E P 1$ reduced resistance to the nematode pathogen Meloidogyne incognita in tobacco. A, Symptoms of UEP1-RNAi plants of line 4 after drenching inoculation with $M$. incognita at second-stage juveniles at 30 days postinfiltration. Arrows indicate root knots. EV = empty vector. B, Disease index analysis for the inoculated plants. Calculation of disease index is based on the 0-to-5 galling index system described in the Materials and Methods. Data represent mean \pm standard error of three independent experiments. Double asterisks $(* *)$ indicate significant difference of disease index between UEP1-RNAi plants and EV control plants $(P<0.01$, $t$ test).
(Backmair et al. 1990). Therefore, the phenotypes of the Ub R48-transgenic plants highly resemble those of the UEPI transgenic tobacco obtained in this study (Figs. 1, 2, and 3), indicating that, in the $U E P 1$ transgenic tobacco plants, the Ub system should have been perturbated.

Tobacco $U E P 1$ modulates disease resistance. Our results demonstrate that UEP1 is widely involved in tobacco disease resistance against a variety of pathogens, including the oomycete $P$. aphanidermatum, viruses TMV and CMV, and nematode $M$. incognita. This is not unexpected considering that $U E P 1$ as a precursor of $\mathrm{Ub}$ may profoundly affect the accumulation level of $\mathrm{Ub}$ and, thus, alter the level of substrate protein ubiquitylation for $26 \mathrm{~S}$ proteasome-dependent protein degradation, which has been reported to be substantially involved in plant resistance against various types of pathogens (Alcaide-Loridan and Jupin 2012; Dielen et al. 2010; Marino et al. 2012; Ustun et al. 2016). Notably, UEP1 differentially regulates resistance to different pathogens: negatively to $P$. aphanidermatum, TMV, and CMV and positively to $M$. incognita. This may reflect the fact that contrast (negative versus positive) regulators of resistance are subjected to degradation by the Ub-26S proteasome system in these different pathosystems. The negative regulation of $U E P 1$ in tobacco resistance against TMV is reminiscent of the previous reports that Ub-transgenic tobacco plants showing cosuppression of endogenous $\mathrm{Ub}$ and Ub R48transgenic tobacco plants manifesting perturbation of Ub-26S proteasome system exhibit increased resistance against this virus (Backmair et al. 1990; Becker et al. 1993). The effect of altered expression levels of plant endogenous $U E P$ on resistance to nematodes has not been previously reported. However, GrUBCEP12, the noncanonical UEP comprising a canonical Ub and CEP12, has been found to be an effector of the potato cyst nematode $G$. rostochiensis, playing important roles in plant parasitism (Chen et al. 2013; Chronis et al. 2013). The Ub moiety seems to affect the host Ub-26S proteasome system, because overexpression of GrUBCEP12 but not GrCEP12 strongly downregulated expression of the host $26 \mathrm{~S}$ proteasome subunit RPN2a (Chronis et al. 2013). However, it is unclear whether and how the total Ub level in planta in GrUBCEP12 overexpression plants changes and, thus, it is not known whether the total Ub level in GrUBCEP12 overexpression plants significantly reduced due to the cosuppression of plant endogenous Ub genes as observed in Ub-transgenic and transiently expressed plants (Becker et al. 1993; Karrer et al. 1998). Determination of Ub level in planta in the GrUBCEP12 overexpression plants will clarify the functional mechanism of the Ub moiety underlying the resistance in these plants. In addition, as far as we know, this is the first report on the role of the $U E P$ gene in plant resistance against an oomycete pathogen.

In addition to the resistance against the pathogens investigated in this study, UEP 1 may also modulate other layers of plant defenses such as PTI and ETI. PTI is initiated by recognition of PAMPs by PRRs, while ETI is initiated by recognition of effectors typically by nucleotide-binding site LRR receptors (NLRs). A list of PRRs and NLRs as well as their signaling components have been reported to be targeted for ubiquitination (Mithoe and Menke 2018; Zhou and Zeng 2017). In this context, given that UEPI is the main source of $\mathrm{Ub}$ and, thus, might affect ubiquitination, it is conceivable that UEP1 might influence PTI and ETI. Identification of PTI and ETI components serving as substrates of $U E P l$ is interesting research that remains to be conducted.

It needs to be mentioned that $U E P 1$ might also be indirectly involved in tobacco resistance. That is, RNAi of UEP1 causes strong cell death in the lower leaves of plants, which might somehow activate resistance in the upper, asymptomatic leaves. In this scenario, resistance to multiple pathogens in UEPI-RNAi transgenic plants resembles systemic acquired resistance and the phenotype of some "lesion mimic" mutants (LMMs) (Bruggeman et al. 2015; Lorrain et al. 2003). The lesions of LMMs resulting 
from the altered regulation of cell death processes in the absence of pathogen attack resemble an HR-mediated cell death which, in some instances, leads to enhanced disease resistance to multiple pathogens due to constitutive accumulation of defense-related hormones and expression of the PR genes (Bruggeman et al. 2015; Lorrain et al. 2003). Meanwhile, mutants that show enhanced resistance to some pathogens while exhibiting increased susceptibility to others have been reported. For example, barley seedlings carrying the mlo mutation showed enhanced resistance to Blumeria graminis f. sp. hordei and Phytophthora palmivora and increased susceptibility to Magnaporthe grisea (Jarosch et al. 1999; Kusch and Panstruga 2017).

Mechanisms underlying tobacco $U E P 1$-mediated modulation of cell death and disease resistance. To reveal the molecular mechanisms underlying UEPl-mediated modulation of cell death and disease resistance, the transcript profile of necrotic tissues of UEP1-RNAi plants was compared with that of non-RNAi control plants through tobacco mRNA microarray analyses, considering that $\mathrm{Ub}$ not only functions through protein degradation but also plays important roles in transcriptional regulation (Conaway et al. 2002). Notably, genes encoding several JAZ proteins, including JAZ1 and JAZ3, the repressors of JA signaling, were upregulated by approximately 5.0- to 11.4-fold, whereas genes coding for a set of EFEs/ACOs, the key enzymes to catalyze the biosynthesis of ET, were downregulated by approximately 2.0 - to

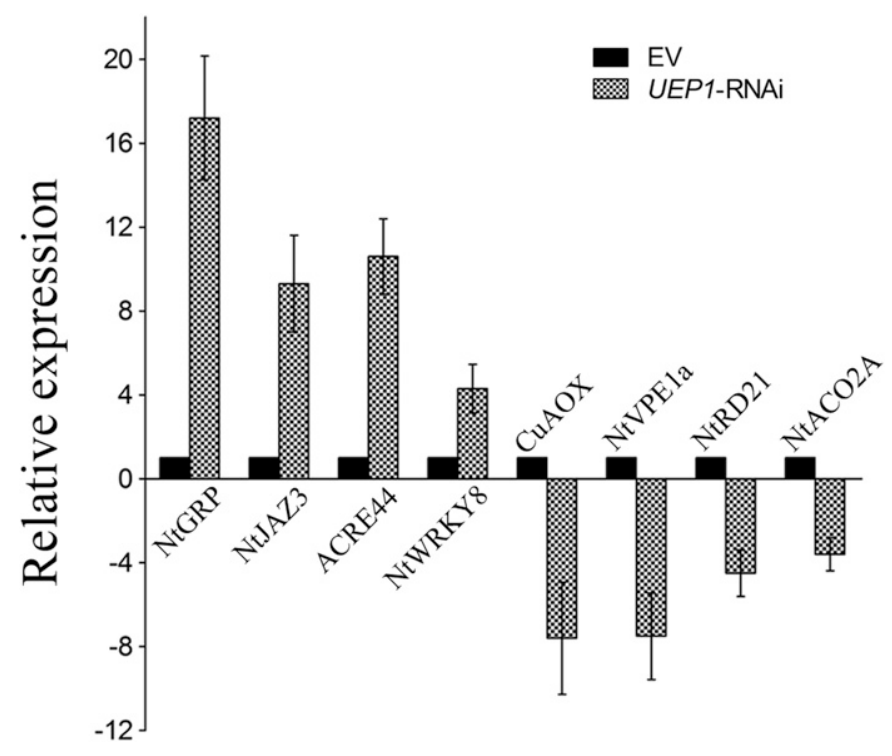

Fig. 8. Expression analyses of eight differentially expressed genes by real-time quantitative polymerase chain reaction. $18 \mathrm{~S}$ ribosomal DNA serves as an inner control. RNAi $=$ RNA interference and EV $=$ empty-vector control. Data represent mean \pm standard error of three independent experiments.
A

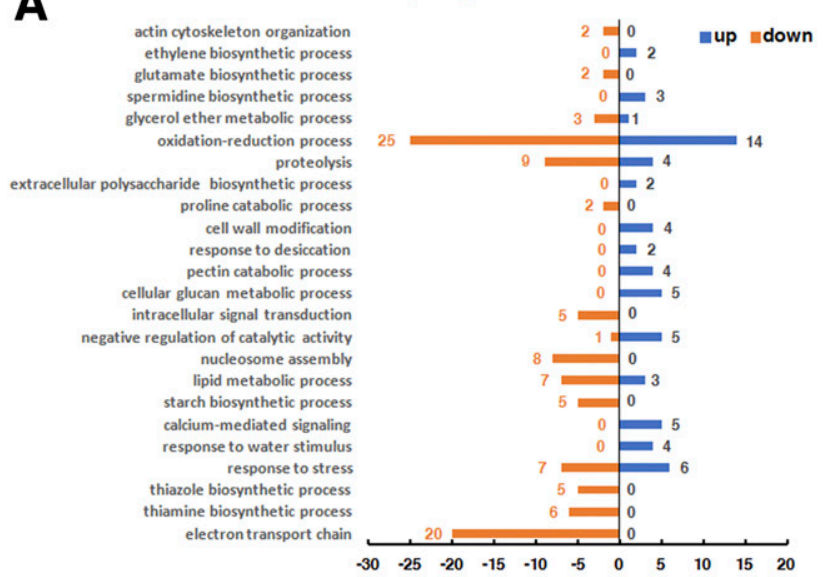

C

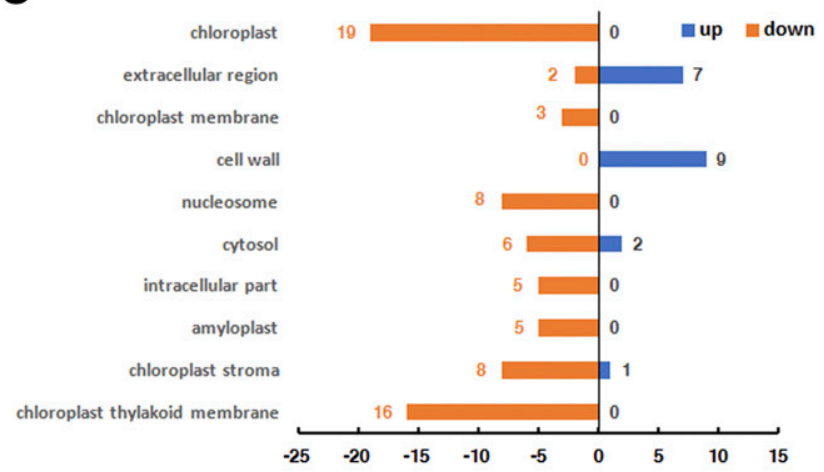

B

Molecular function

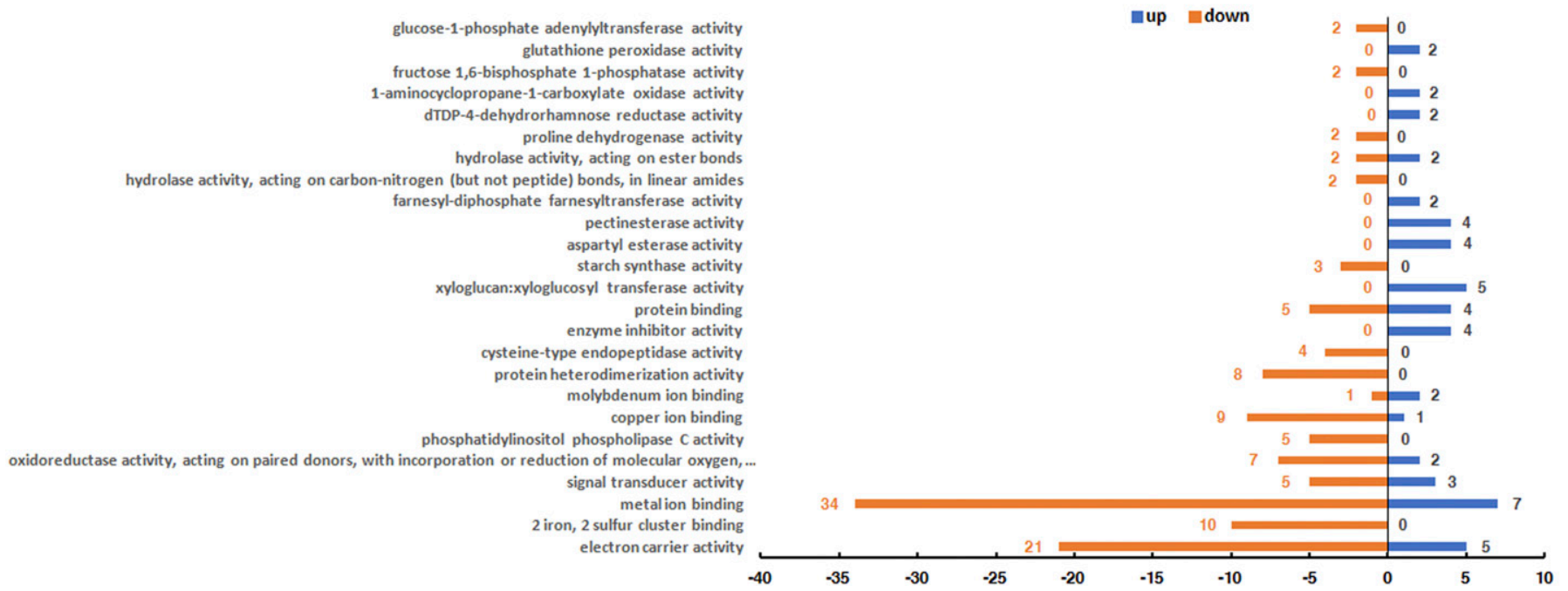

Fig. 7. Gene ontology (GO) terms for genes differentially expressed between necrotic tissues of UEPI-RNA interference (RNAi) line 4 and healthy tissues of empty-vector (EV) control plants. Only statistically significant $(P<0.05, t$ test) $\mathrm{GO}$ terms associated with $\mathbf{A}$, biological process; $\mathbf{B}$, molecular function; and $\mathbf{C}$, cellular component were presented. Numbers of upregulated and downregulated genes for each GO term are indicated. 
3.3-fold in UEP1-RNAi plants, indicating that both JA and ET pathways were strongly suppressed in UEPl-RNAi plants (Table 1). Intriguingly, JAZ proteins are targets of the Ub-26S proteasome system and are rapidly degraded in the presence of JA, and JAZ genes are transcriptionally upregulated by JA (Chini et al. 2007; Thines et al. 2007). The dramatic upregulation of JAZ genes likely leads to accumulation of JAZ proteins, which likely can be attributed to the suppression of JAZ protein proteolysis by Ub-26S proteasome system caused by cosuppression of Ub genes such as UBQ3 and UBQ10 in UEP1-RNAi plants due to their high sequence homology with the UEPI gene (Table 1). Interestingly, JA has been reported to play vital roles in basal resistance to root-knot nematodes (Zhou et al. 2015). Thus, the suppression of the JA pathway in UEPI-RNAi plants might be the cause of the reduced resistance to Meloidogyne incognita (Fig. 6). In addition, the suppression of JA and ET pathways is indirectly confirmed by the promotion of the salicylic acid (SA) pathway, as manifested by the strong upregulation of gene $P R 1$, the molecular marker of the SA pathway (Table 1), regarding a mutually antagonistic interaction between SA and JA or ET signaling pathways (An and Mou 2011). It is well known that SA plays crucial roles in resistance against various pathogens, including viruses and oomycetes (An and Mou 2011; Vlot et al. 2009). Therefore, promotion of the SA pathway may contribute to the enhanced resistance to Pythium aphanidermatum, TMV, and CMV in the UEP1-RNAi plants (Figs. 4 and 5).

Expressional alteration of genes encoding VPE, RD21, and ATG8 may be correlated with cell death and disease resistance in
UEP1-RNAi plants. VPEs are involved in stimuli-elicited cell death (Kuroyanagi et al. 2005; Rojo et al. 2004), developmentally regulated programmed cell death (Teper-Bamnolker et al. 2017), and disease resistance against various pathogens, including Pseudomonas syringae, Botrytis cinerea, and Turnip mosaic virus (Rojo et al. 2004). In particular, VPE $\gamma$ exhibits caspase-like activity and, thus, contributes to HR and resistance (Rojo et al. 2004). RD21, like VPEs, is also a vacuolar protease. RD21 and its homologs play important roles in pathogen-induced cell death, disease resistance against necrotroph pathogens B. cinerea and Sclerotinia sclerotiorum (Lampl et al. 2013; Shindo et al. 2012), and plant development (Rustgi et al. 2017). ATG8 is a core component of autophagy, and autophagy has been increasingly and widely linked to cell death, HR, and disease resistance (Zhou et al. 2014). The roles of autophagy in cell death, HR, and disease resistance are complex, depending on the stimuli and pathosystems. For example, autophagy can play a positive role in plant antiviral defense by targeting viruses and viral RNAi suppressors for degradation, whereas it can also be hijacked by viral RNAi suppressors for the degradation of critical components of the host cell RNAi machinery (Han et al. 2015; Haxim et al. 2017; Zhou et al. 2014). Interestingly, there are complex crosstalk and interplay between the Ub-26S proteasome system and autophagy (Ji and Kwon 2017). In this study, we found that UEP 1 plays distinct roles in resistance against different pathogens: negatively against oomycete Pythium aphanidermatum and viruses TMV and CMV, and positively to nematode M. incognita. In UEP1-RNAi plants, VPE, RD21, and ATG8 all are significantly downregulated (Table 1), which might be partially

TABLE 1. Selected differentially expressed genes in comparison between UEP1-RNA interference (RNAi) and empty-vector (EV) control plants

\begin{tabular}{|c|c|c|c|}
\hline Probe ID & $\begin{array}{l}\text { Corresponding expressed } \\
\text { sequence tag sequence }\end{array}$ & Description & $\begin{array}{c}\text { Fold change } \\
(U E P-\mathrm{RNAi} / \mathrm{EV})\end{array}$ \\
\hline A_95_P103367 & NtA.2985 & Glycine-rich protein precursor & 21.6 \\
\hline A_95_P104267 & NtA.2985 & Glycine-rich protein precursor & 16.9 \\
\hline A_95_P125502 & NtA.2985 & Glycine-rich protein precursor & 10.6 \\
\hline A_95_P103757 & NtA.2985 & Glycine-rich protein precursor & 9.5 \\
\hline A_95_P000611 & NtA.26956 & Glycine-rich protein precursor & 7.2 \\
\hline A_95_P000056 & NtA.2714 & Glycine-rich protein precursor & 6.8 \\
\hline A_95_P158972 & NtA.2714 & Glycine-rich protein precursor & 6.6 \\
\hline A_95_P164682 & NtA.2985 & Glycine-rich protein precursor & 6.1 \\
\hline A_95_P164872 & NtA.2985 & Glycine-rich protein precursor & 6 \\
\hline A_95_P104387 & NtA.2714 & Glycine-rich protein precursor & 5.3 \\
\hline A_95_P109447 & NtA.2714 & Glycine-rich protein precursor & 4.9 \\
\hline A_95_P297578 & NtA.12079 & Cf-9/Avr9 rapidly elicited protein 44 (ACRE44) & 12.6 \\
\hline A_95_P234579 & NtA.2780 & Jasmonate ZIM-domain protein 3 & 11.4 \\
\hline A_95_P022406 & NtA.2780 & Jasmonate ZIM-domain protein 3 & 10.5 \\
\hline A_95_P104317 & NtA.2780 & Jasmonate ZIM-domain protein 3 & 9.9 \\
\hline A_95_P141952 & NtA.22914 & Jasmonate ZIM-domain protein 3 & 9.4 \\
\hline A_95_p293653 & NtA.16863 & Jasmonate-ZIM-domain protein 1 & 8 \\
\hline A_95_p159272 & NtA.22914 & Jasmonate-ZIM-domain protein 1 & 5 \\
\hline A_95_P163382 & NtA.6418 & S1GRAS2 & 8.6 \\
\hline A_95_P063575 & NtA.21822 & Leucine-rich repeat family protein & 8.4 \\
\hline A_95_P293048 & NtA.9459 & Xyloglucosyl transferase & 6.3 \\
\hline A_95_P121337 & NtA.9459 & Xyloglucosyl transferase & 6.1 \\
\hline A_95_P258081 & NtA.9459 & Xyloglucosyl transferase & 5.7 \\
\hline A_95_P112902 & NtA.9459 & Xyloglucosyl transferase & 4.8 \\
\hline A_95_P158797 & NtA.6400 & Carboxylesterase & 5.8 \\
\hline A_95_P183507 & NtA.6400 & Carboxylesterase & 5.7 \\
\hline A_95_P164752 & NtA.9510 & NtERD10C & 5.7 \\
\hline A_95_P028846 & NtA.9510 & NtERD10C & 4.7 \\
\hline A_95_p195052 & NtA. 8125 & AtCPK28 & 3.7 \\
\hline A_95_p021516 & NtA. 2727 & NtCAM7 & 3.2 \\
\hline A_95_p258231 & NtA.7696 & AtCAM3 & 2.7 \\
\hline A_95_p291478 & $\ldots$ & AtCDPK6 & 2.4 \\
\hline A_95_p034853 & NtA.11596 & AtCDPK32 & 2.2 \\
\hline A_95_p129262 & NtA.972 & AtCIPK3 & 2.1 \\
\hline A_95_P287768 & NtA.15675 & AtWRKY31 & 6.3 \\
\hline A_95_p161782 & NtA.9092 & AtWRKY31 & 2.3 \\
\hline A_95_p162582 & NtA.9092 & AtWRKY6 & 3.8 \\
\hline
\end{tabular}


responsible for the constitutive cell death and disease resistance in these plants against some pathogens checked in this study. The detailed mechanisms for how these genes were regulated by UEP1 and how they function in modulating cell death and disease resistance in UEP1-RNAi plants await further dissection.

$\mathrm{A} \mathrm{CuAO}$ gene was downregulated by 11.3 -fold and is the most downregulated gene of the DE genes in UEP1-RNAi plants. CuAOs play important roles in production of active species such as $\mathrm{H}_{2} \mathrm{O}_{2}$ and $\mathrm{NO}$ and, therefore, are involved in regulation of development and defense (Angelini et al. 2010; Cona et al. 2006; Groß et al. 2017; Wimalasekera et al. 2011).

The most upregulated genes in UEPI-RNAi plants encoded a set of GRPs, which were detected by 11 probes, including the one whose expression is upregulated by 21.6 -fold. Numerous GRPs are components of the plant cell wall. They are accumulated in the vascular tissues. Their expression is regulated developmentally and by external stimuli ranging from pathogen infection to environmental stresses (Mangeon et al. 2010; Mousavi and Hotta 2005). Thus, it is possible that the strong upregulation of GRPs in UEP1-RNAi plants somehow affects leaf development and plays a positive role in defense through reinforcing the cell wall by enhancing cell wall components, lignification, and callose deposition. In addition, some RNAbinding GRPs have been reported to regulate cell death, defense, and development (Mangeon et al. 2010). For instance, Arabidopsis glycine-rich RNA-binding protein7 (AtGRP7) is involved in defense against a wide range of pathogens. Interestingly, AtGRP7 enhances resistance to TMV (Lee et al. 2012). This fits our observation that UEP1-RNAi plants show high levels of GRP gene expression and increased resistance to TMV. A pepper glycine-rich RNA-binding protein (CaGRP1) was found to interact with receptor-like cytoplasmic protein kinase 1 (CaPIK1) and negatively regulates CaPIK1-triggered cell death and defense responses by suppressing ROS accumulation (Kim et al. 2015). Intriguingly, a Lilium GRP LsGRP1 was ubiquitylated and induced by SA treatment and pathogen infection (Lin and Chen 2014). This indicates that the increased accumulation of GRPs in UEP1-RNAi plants might be directly caused by the reduction of Ub-26S proteasome system-mediated GRP protein degradation due to less accumulation of $\mathrm{Ub}$ resulting from the silencing of UEP1 and consequent drop of GRP protein ubiquitylation. It would be worthwhile to identify whether the DE GRPs in this study are, indeed, targets of the Ub-26S proteasome system.

Conclusions. NbUEP1 is a Ub-S27a-type $U E P$ gene from $N$. benthamiana. Both VIGS and stable RNAi plant assays demonstrated that silencing of $N b U E P 1$ caused constitutive cell death. RNAi of $N b U E P 1$ enhanced resistance to oomycete $P$. aphanidermatum and viruses TMV and CMV, whereas it alleviated resistance to nematode $M$. incognita. Transcription profiling analysis revealed some potential pathways and regulators of $N b U E P 1$-mediated cell death and resistance. These included JA

TABLE 1. (Continued from previous page)

\begin{tabular}{|c|c|c|c|}
\hline Probe ID & $\begin{array}{l}\text { Corresponding expressed } \\
\text { sequence tag sequence }\end{array}$ & Description & $\begin{array}{c}\text { Fold change } \\
(U E P-\mathrm{RNAi} / \mathrm{EV})\end{array}$ \\
\hline A_95_p032421 & NtA.4132 & AtWRKY33 & 2.8 \\
\hline A_95_p010511 & $\ldots$ & AtWRKY40 & 2.3 \\
\hline A_95_p034843 & NtA.17662 & Peroxidase 12 (PER12) & 4.3 \\
\hline A_95_p021026 & $\ldots$ & AtTH9 (thioredoxin H-type 9) & 4.1 \\
\hline A_95_p250327 & NtA.7214 & AtTH9 (thioredoxin H-type 9) & 2.7 \\
\hline A_95_P291458 & NtA.16419 & Copper amine oxidase family protein & -11.3 \\
\hline A_95_P269472 & NtA.7223 & AtGSR1 & -8.1 \\
\hline A_95_P250807 & NtA.7321 & Vacuolar processing enzyme & -7.5 \\
\hline A_95_P163492 & NtA.4071 & Vacuolar processing enzyme & -6.9 \\
\hline A_95_P007441 & NtA.4071 & Vacuolar processing enzyme & -6.7 \\
\hline A_95_P239420 & NtA.4048 & Proline oxidase & -7.5 \\
\hline A_95_P218312 & NtA.11690 & Serine carboxypeptidase (Arabidopsis thaliana) & -6.2 \\
\hline A_95_P255594 & NtA.8817 & Serine carboxypeptidase (A. thaliana) & -5.6 \\
\hline A_95_P286283 & NtA.15368 & Bundle sheath-defective protein 2 family & -5 \\
\hline A_95_P029006 & NtA. 15368 & Bundle sheath-defective protein 2 family & -4.7 \\
\hline A_95_P121002 & NtA.6396 & AtGLDP1 (glycine decarboxylase P 1) & -4.9 \\
\hline A_95_P247197 & NtA.6396 & AtGLDP1 (glycine decarboxylase P 1) & -4.4 \\
\hline A_95_P103707 & NtA.7100 & NtPLA2 (phospholipase A2) & -4.5 \\
\hline A_95_P292133 & NtA.2590 & AtRD21 (responsive to dehydration 21) & -4.3 \\
\hline A_95_P227359 & NtA.954 & Glycerate dehydrogenase & -4.2 \\
\hline A_95_P129652 & NtA.954 & Glycerate dehydrogenase & -4 \\
\hline A_95_P021996 & NtA.8612 & Phosphoinositide binding & -4.2 \\
\hline A_95_P279283 & NtA.13943 & AtFRO6 (ferric reduction oxidase 6) & -4.1 \\
\hline A_95_p150732 & NtA.8560 & Peptidase S41 family protein & -4.5 \\
\hline A_95_p045706 & NtA. 8560 & Peptidase S41 family protein & -3.5 \\
\hline A_95_p010991 & NtA.7063 & AtEFE (ethylene-forming enzyme) & -3.3 \\
\hline A_95_p008226 & NtA.7063 & AtEFE (ethylene-forming enzyme) & -2.8 \\
\hline A_95_p179962 & $\ldots$ & AtEFE (ethylene-forming enzyme) & -2.6 \\
\hline A_95_p130452 & NtA. 10728 & AtEFE (ethylene-forming enzyme) & -2.4 \\
\hline A_95_p156612 & NtA.7064 & AtEFE (ethylene-forming enzyme) & -2.3 \\
\hline A_95_p020477 & NtA.7063 & AtEFE (ethylene-forming enzyme) & -2.2 \\
\hline A_95_p156027 & NtA.7063 & AtEFE (ethylene-forming enzyme) & -2.2 \\
\hline A_95_p179957 & NtA. 10728 & AtEFE (ethylene-forming enzyme) & -2 \\
\hline A_95_p227174 & NtA.911 & C3HC4-type zinc-finger family protein & -2.9 \\
\hline A_95_p014336 & NtA.9486 & AtUBQ3 & -2.7 \\
\hline A_95_p112762 & NtA.9486 & AtUBQ3 & -2.4 \\
\hline A_95_p010476 & NtA.9486 & AtUBQ3 & -2.2 \\
\hline A_95_p012331 & NtA.22166 & AtUBQ10 & -2.1 \\
\hline A_95_p131717 & NtA.881 & ATG8c (autophagy 8c) & -2.5 \\
\hline A_95_p024721 & NtA.2797 & ATG8c (autophagy 8c) & -2 \\
\hline
\end{tabular}


and ET pathways, lipid metabolism, CuAO-mediated active species generation, GRPs, VPE, RD21, and autophagy.

\section{LITERATURE CITED}

Alcaide-Loridan, C., and Jupin, I. 2012. Ubiquitin and plant viruses, let's play together. Plant Physiol. 160:72-82.

An, C., and Mou, Z. 2011. Salicylic acid and its function in plant immunity. J. Integr. Plant Biol. 53:412-428.

Angelini, R., Cona, A., Federico, R., Fincato, P., Tavladoraki, P., and Tisi, A. 2010. Plant amine oxidases 'on the move': An update. Plant Physiol. Biochem. 48:560-564.

Bachmair, A., Becker, F., Masterson, R., and Schell, J. 1990. Perturbation of the ubiquitin system causes leaf curling, vascular tissue alterations and necrotic lesions in a higher plant. EMBO J. 9:4543-4549.

Barker, K. R. 1985. Nematode extraction and bioassays. Pages 19-35 in: An Advanced Treatise on Meloidogyne,Vol. II. Methodology. K. R. Barker, C. C. Carter, and J. N. Sasser, eds. North Carolina State University Graphics, Raleigh, NC, U.S.A.

Becker, F., Buschfeld, E., Schell, J., and Bachmair, A. 1993. Altered response to viral infection by tobacco plants perturbed in ubiquitin system. Plant J. 3: $875-881$

Bruggeman, Q., Raynaud, C., Benhamed, M., and Delarue, M. 2015. To die or not to die? Lessons from lesion mimic mutants. Front. Plant Sci. 6:24.

Cai, X. Z., Zhou, X., Xu, Y. P., Joosten, M. H. A. J., and de Wit, P. J. G. M. 2007. Cladosporium fulvum CfHNNI1 induces hypersensitive necrosis, defence gene expression and disease resistance in both host and nonhost plants. Plant Mol. Biol. 64:89-101.

Callis, J., Carpenter, T., Sun, C. W., and Vierstra, R. D. 1995. Structure and evolution of genes encoding polyubiquitin and ubiquitin-like proteins in Arabidopsis thaliana ecotype Columbia. Genetics 139:921-939.

Callis, J., Raasch, J. A., and Vierstra, R. D. 1990. Ubiquitin extension proteins of Arabidopsis thaliana. Structure, localization, and expression of their promoters in transgenic tobacco. J. Biol. Chem. 265:12486-12493.

Cao, J. Y., Xu, Y. P., Li, W., Li, S. S., Rahman, H., and Cai, X. Z. 2016. Genome-wide identification of Dicer-like, Argonaute and RNA-dependent RNA polymerase gene families in Brassica species and functional analyses of their Arabidopsis homologs in resistance to Sclerotinia sclerotiorum. Front. Plant Sci. 7:1614.

Chen, S., Chronis, D., and Wang, X. 2013. The novel GrCEP12 peptide from the plant-parasitic nematode Globodera rostochiensis suppresses flg22mediated PTI. Plant Signaling Behav. 8:e25359.

Chini, A., Fonseca, S., Fernandez, G., Adie, B., Chico, J. M., Lorenzo, O., García-Casado, G., López-Vidriero, I., Lozano, F. M., Ponce, M. R., Micol, J. L., and Solano, R. 2007. The JAZ family of repressors is the missing link in jasmonate signalling. Nature 448:666-671.

Chronis, D., Chen, S., Lu, S., Hewezi, T., Carpenter, S. C., Loria, R., Baum, T. J., and Wang, X. 2013. A ubiquitin carboxyl extension protein secreted from a plant-parasitic nematode Globodera rostochiensis is cleaved in planta to promote plant parasitism. Plant J. 74:185-196.

Cona, A., Rea, G., Angelini, R., Federico, R., and Tavladoraki, P. 2006. Functions of amine oxidases in plant development and defence. Trends Plant Sci. 11:80-88.

Conaway, R. C., Brower, C. S., and Conaway, J. W. 2002. Emerging roles of ubiquitin in transcription regulation. Science 296:1254-1258.

Dielen, A. S., Badaoui, S., Candresse, T., and German-Retana, S. 2010. The ubiquitin/26S proteasome system in plant-pathogen interactions: A neverending hide-and-seek game. Mol. Plant Pathol. 11:293-308.

Du, Z., Zhou, X., Ling, Y., Zhang, Z., and Su, Z. 2010. agriGO: A GO analysis toolkit for the agricultural community. Nucleic Acids Res. 38: W64-W70.

Finley, D., Bartel, B., and Varshavsky, A. 1989. The tails of ubiquitin precursors are ribosomal proteins whose fusion to ubiquitin facilitates ribosome biogenesis. Nature 338:394-401.

Groß, F., Rudolf, E. E., Thiele, B., Durner, J., and Astier, J. 2017. Copper amine oxidase 8 regulates arginine-dependent nitric oxide production in Arabidopsis thaliana. J. Exp. Bot. 68:2149-2162.

Han, S., Wang, Y., Zheng, X., Jia, Q., Zhao, J., Bai, F., Hong, Y., and Liu, Y. 2015. Cytoplastic glyceraldehyde-3-phosphate dehydrogenases interact with ATG3 to negatively regulate autophagy and immunity in Nicotiana benthamiana. Plant Cell 27:1316-1331.

Hanania, U., Velcheva, M., Sahar, N., Flaishman, M., Or, E., Dgani, O., and Perl, A. 2009. Suppression and overexpression of ubiquitin extension protein S27a affects cell proliferation and in vitro regeneration in Nicotiana benthamiana. Plant Sci. 176:566-574.

Haxim, Y., Ismayil, A., Jia, Q., Wan, Y., Zheng, X., Chen, T., Qian, L., Liu, N., Wang, Y., Han, S., Cheng, J., Qi, Y., Hong, Y., and Liu, Y. 2017. Autophagy functions as an antiviral mechanism against geminiviruses in plants. eLife $6: \mathrm{e} 23897$.
Jarosch, B., Kogel, K.-H., and Schaffrath, U. 1999. The ambivalence of the barley Mlo locus: Mutations conferring resistance against powdery mildew (Blumeria graminis f. sp. hordei) enhance susceptibility to the rice blast fungus Magnaporthe grisea. Mol. Plant-Microbe Interact. 12: 508-514.

Ji, C. H., and Kwon, Y. T. 2017. Crosstalk and interplay between the ubiquitinproteasome system and autophagy. Mol. Cells 40:441-449.

Karrer, E. E., Beachy, R. N., and Holt, C. A. 1998. Cloning of tobacco genes that elicit the hypersensitive response. Plant Mol. Biol. 36:681-690.

Kim, D. S., Kim, N. H., and Hwang, B. K. 2015. GLYCINE-RICH RNABINDING PROTEIN 1 interacts with RECEPTOR-LIKE CYTOPLASMIC PROTEIN KINASE 1 and suppresses cell death and defense responses in pepper (Capsicum annиum). New Phytol. 205:786-800.

Kuroyanagi, M., Yamada, K., Hatsugai, N., Kondo, M., Nishimura, M., and Hara-Nishimura, I. 2005. Vacuolar processing enzyme is essential for mycotoxin-induced cell death in Arabidopsis thaliana. J. Biol. Chem. 280: 32914-32920.

Kusch, S., and Panstruga, R. 2017. mlo-based resistance: An apparently universal "weapon" to defeat powdery mildew disease. Mol. Plant-Microbe Interact. 30:179-189.

Lampl, N., Alkan, N., Davydov, O., and Fluhr, R. 2013. Set-point control of RD21 protease activity by AtSerpin 1 controls cell death in Arabidopsis. Plant J. 74:498-510.

Lee, H. J., Kim, J. S., Yoo, S. J., Kang, E. Y., Han, S. H., Yang, K. Y., Kim, Y. C., McSpadden Gardener, B., and Kang, H. 2012. Different roles of glycine-rich RNA-binding protein 7 in plant defense against Pectobacterium carotovorum, Botrytis cinerea, and tobacco mosaic viruses. Plant Physiol. Biochem. 60:46-52.

Li, W., Xu, Y. P., Zhang, Z. X., Cao, W. Y., Li, F., Zhou, X., Chen, G. Y., and Cai, X. Z. 2012. Identification of genes required for nonhost resistance to Xanthomonas oryzae pv. oryzae reveals novel signaling components. PLoS One 7:e42796.

Lin, C. H., and Chen, C. Y. 2014. Characterization of the dual subcellular localization of Lilium LsGRP1, a plant class II glycine-rich protein. Phytopathology 104:1012-1020.

Lorrain, S., Vailleau, F., Balague, C., and Roby, D. 2003. Lesion mimic mutants: Keys for deciphering cell death and defense pathways in plants? Trends Plant Sci. 8:263-271.

Mangeon, A., Junqueira, R. M., and Sachetto-Martins, G. 2010. Functional diversity of the plant glycine-rich proteins superfamily. Plant Signaling Behav. 5:99-104.

Marino, D., Peeters, N., and Rivas, S. 2012. Ubiquitination during plant immune signaling. Plant Physiol. 160:15-27.

Mithoe, S. C., and Menke, F. L. H. 2018. Regulation of pattern recognition receptor signalling by phosphorylation and ubiquitination. Curr. Opin. Plant Biol. 45:162-170

Mousavi, A., and Hotta, Y. 2005. Glycine-rich proteins: A class of novel proteins. Appl. Biochem. Biotechnol. 120:169-174.

Nicholas, K. B., Nicholas, H., and Deerfield, D. 1997. GeneDoc: Analysis and visualization of genetic variation. Embnew News 4.

Redman, K. L., and Rechsteiner, M. 1989. Identification of the long ubiquitin extension as ribosomal protein S27a. Nature 338:438-440.

Rojo, E., Martin, R., Carter, C., Zouhar, J., Pan, S., Plotnikova, J., Jin, H., Paneque, M., Sánchez-Serrano, J. J., Baker, B., Ausubel, F. M., and Raikhel, N. V. 2004. VPE $\gamma$ exhibits a caspase-like activity that contributes to defense against pathogens. Curr. Biol. 14:1897-1906.

Rustgi, S., Boex-Fontvieille, E., Reinbothe, C., von Wettstein, D., and Reinbothe, S. 2017. Serpin1 and WSCP differentially regulate the activity of the cysteine protease RD21 during plant development in Arabidopsis thaliana. Proc. Natl. Acad. Sci. U.S.A. 114:2212-2217.

Shindo, T., Misas-Villamil, J. C., Hörger, A. C., Song, J., and van der Hoorn, R. A. 2012. A role in immunity for Arabidopsis cysteine protease RD21, the ortholog of the tomato immune protease C14. PLoS One 7:e29317.

Spence, J., Gali, R. R., Dittmar, G., Sherman, F., Karin, M., and Finley, D. 2000. Cell cycle-regulated modification of the ribosome by a variant multiubiquitin chain. Cell 102:67-76.

Teper-Bamnolker, P., Buskila, Y., Belausov, E., Wolf, D., Doron-Faigenboim, A., Ben-Dor, S., Van der Hoorn, R. A. L., Lers, A., and Eshel, D. 2017. Vacuolar processing enzyme activates programmed cell death in the apical meristem inducing loss of apical dominance. Plant Cell Environ. 40:2381-2392.

Thines, B., Katsir, L., Melotto, M., Niu, Y., Mandaokar, A., Liu, G., Nomura, K., He, S. Y., Howe, G. A., and Browse, J. 2007. JAZ repressor proteins are targets of the SCFCOI1 complex during jasmonate signalling. Nature 448: 661-665.

Ustun, S., Sheikh, A., Gimenez-Ibanez, S., Jones, A., Ntoukakis, V., and Bornke, F. 2016. The proteasome acts as a hub for plant immunity and is targeted by Pseudomonas type III effectors. Plant Physiol. 172:1941-1958.

Vierstra, R. D. 2012. The expanding universe of ubiquitin and ubiquitin-like modifiers. Plant Physiol. 160:2-14. 
Vlot, A. C., Dempsey, D. A., and Klessig, D. F. 2009. Salicylic acid, a multifaceted hormone to combat disease. Annu. Rev. Phytopathol. 47:177-206.

Wimalasekera, R., Villar, C., Begum, T., and Scherer, G. F. 2011. COPPER AMINE OXIDASE1 (CuAO1) of Arabidopsis thaliana contributes to abscisic acid- and polyamine-induced nitric oxide biosynthesis and abscisic acid signal transduction. Mol. Plant 4:663-678.

Yan, N., Doelling, J. H., Falbel, T. G., Durski, A. M., and Vierstra, R. D. 2000. The ubiquitin-specific protease family from Arabidopsis. AtUBP1 and 2 are required for the resistance to the amino acid analog canavanine. Plant Physiol. 124:1828-1843.
Zhao, Y., Liu, W., Xu, Y. P., Cao, J. Y., Braam, J., and Cai, X. Z. 2013. Genome-wide identification and functional analyses of calmodulin genes in Solanaceous species. BMC Plant Biol. 13:70.

Zhou, B., and Zeng, L. 2017. Conventional and unconventional ubiquitination in plant immunity. Mol. Plant Pathol. 18:1313-1330.

Zhou, J., Jia, F., Shao, S., Zhang, H., Li, G., Xia, X., Zhou, Y., Yu, J., and Shi, K. 2015. Involvement of nitric oxide in the jasmonate-dependent basal defense against root-knot nematode in tomato plants. Front. Plant Sci. 6:193.

Zhou, J., Yu, J. Q., and Chen, Z. 2014. The perplexing role of autophagy in plant innate immune responses. Mol. Plant Pathol. 15:637-645. 